\title{
A Forward Genetic Screen in Sclerotinia sclerotiorum Revealed the Transcriptional Regulation of Its Sclerotial Melanization Pathway
}

\author{
Yan Xu, ${ }^{1,2}$ Kevin Ao, ${ }^{1,2}$ Lei Tian, ${ }^{1,2}$ Yilan Qiu, ${ }^{3}$ Xingchuan Huang, ${ }^{4}$ Xueru Liu, ${ }^{1,2}$ Ryan Hoy, ${ }^{1,2}$ \\ Yishan Zhang, ${ }^{1}$ Khalid Youssef Rashid, ${ }^{5}$ Shitou Xia, ${ }^{4}$ and Xin $\mathrm{Li}^{1,2, \dagger}$ \\ ${ }^{1}$ Michael Smith Laboratories, University of British Columbia, Vancouver, BC V6T 1Z4, Canada \\ ${ }^{2}$ Department of Botany, University of British Columbia, Vancouver, BC V6T 1Z4, Canada \\ ${ }^{3}$ Department of Life Science, Hunan Normal University, Changsha 410081, China \\ ${ }^{4}$ Hunan Provincial Key Laboratory of Phytohormones and Growth Development, College of Bioscience and Biotechnology, \\ Hunan Agricultural University, Changsha 410128, China \\ ${ }^{5}$ Oilseed Crops Pathology, Science and Technology Branch, Ottawa Research and Development Centre, K.W. Neatby \\ Building, Agriculture and Agri-Food Canada, Ottawa K1A 0C6, Canada
}

Accepted 22 November 2021.

\begin{abstract}
Most plant fungal pathogens that cause worldwide crop losses are understudied, due to various technical challenges. With the increasing availability of sequenced whole genomes of these non-model fungi, effective genetic analysis methods are highly desirable. Here, we describe a newly developed pipeline, which combines forward genetic screening with high-throughput next-generation sequencing to enable quick gene discovery. We applied this pipeline in the notorious soilborne phytopathogen Sclerotinia sclerotiorum and identified 32 mutants with various developmental and growth deficiencies. Detailed molecular studies of three melanizationdeficient mutants provide a proof of concept for the effectiveness of our method. A master transcription factor was found to regulate melanization of sclerotia through the DHN (1,8-dihydroxynaphthalene) melanin biosynthesis pathway. In addition, these mutants revealed that sclerotial melanization is important for sclerotia survival under abiotic stresses, sclerotial surface structure, and sexual reproduction. Foreseeably, this pipeline can be applied to facilitate efficient in-depth studies of other non-model fungal species in the future.
\end{abstract}

\section{${ }^{\dagger}$ Corresponding author: X. Li; xinli@msl.ubc.ca}

Funding: This work was financially supported by Canada Foundation for Innovation John R. Evans Leaders Fund, Natural Sciences and Engineering Research Council of Canada Discovery grant and CREATE (PRoTECT) programs. K. Ao is partly supported by the Alexander Graham Bell Canada Graduate Scholarships doctoral program and the University of British Columbia Four-Year Fellowship program. Y. Xu and L. Tian are partly supported by scholarships from the Chinese Scholarship Council.

*The $\boldsymbol{e}$-Xtra logo stands for "electronic extra" and indicates there are supplementary figures and tables published online.

The author(s) declare no conflict of interest. distributed under the CC BY 4.0 International license.
Keywords: DHN melanin, forward genetic analysis, next-generation sequencing, Sclerotinia sclerotiorum, sclerotial formation, soilborne phytopathogen, SsSMRI

Plant diseases are major threats to agriculture (Sturz et al. 1997), accounting for losses of at least $10 \%$ of the annual global harvest (Strange and Scott 2005). Among microbial pathogens, fungi represent the most diverse group of ecologically and economically relevant threats (Doehlemann et al. 1987), often causing catastrophes in the field and in postharvest products (Fisher et al. 2012; Fry 2008; Ullstrup 1972; Williamson et al. 2007). Despite the damage they inflict to crops, most plant fungal pathogens and, indeed, fungi in general are understudied. This is due to various difficulties, including culturability, lack of highquality whole-genome sequences, multinuclear somatic inheritance patterns, and physical or chemical barriers inhibiting molecular biology experiments.

With the advent of modern high-throughput sequencing technologies, the number of fully sequenced fungal genomes is rapidly growing, and many belonging to species that have never been studied before. The availability of these sequenced genomes for numerous plant fungal pathogens is enabling reverse genetic analysis. Efficient targeted gene knockout methods are being developed for many of these understudied organisms. Some examples include homologous recombination protocols for gene replacement, RNA interference-mediated gene silencing, Agrobacterium-mediated insertional mutagenesis, and CRISPR-based gene editing (Bhadauria et al. 2009; Mullins and Kang 2001). Despite the many findings that reverse genetics studies have produced, it is inevitably limited by bias. Efficient forward genetic approaches are therefore needed for unbiased gene discovery.

Sclerotinia sclerotiorum (Lib.) de Bary is considered one of the most notorious soilborne fungal pathogens. This ascomycete has an extremely broad host range of over 600 plant species of mostly dicotyledonous and some monocotyledonous plants (Boland and Hall 1994; Liang and Rollins 2018). Major features of its development include the production of a vegetative resting structure, termed sclerotium, and a reproductive apparatus, named apothecium (Willetts and Wong 1980). Sclerotia can germinate myceliogenically to infect adjacent plants and 
carpogenically to spread ascospores in the air. Their highlymelanized outer surface has been hypothesized to be responsible for their long-term survival in soil, rendering this pathogen extremely hard to control in the field (Butler et al. 2009). Although many reverse genetics studies have been carried out on this fungus (Xia et al. 2020), only a couple of low efficiency mutant screens were attempted using UV irradiation or Agrobacterium-mediated T-DNA insertional mutagenesis (Godoy et al. 1990; Miller et al. 1989; Xu and Chen 2013). In addition, the lack of asexual conidia spores and the multinuclear nature of the vegetative hyphae (Willetts and Wong 1980) of S. sclerotiorum also restrict the application of forward genetic methods. Thus, more efficient forward genetic methods are highly desirable.

Here, we describe an original high-throughput forward genetics pipeline for $S$. sclerotiorum. Using haploid, binucleate ascospores for mutagenesis, mutants defective in sclerotia formation were isolated. Next-generation sequencing (NGS) allowed us to identify the responsible candidate genes quickly. Using three sclerotial melanization-deficient mutants found from the screen, we demonstrate the feasibility of the pipeline for fast gene discovery. Considering its high efficiency, this pipeline has the potential to be broadly applied to other understudied fungi.

\section{RESULTS}

\section{Development of a high-throughput \\ forward genetics pipeline for $\mathbf{S}$. sclerotiorum.}

Since the first public release of the whole genome sequence of strain S. sclerotiorum 1980 in 2011, a growing number of reverse genetic methods have been developed to study this notorious pathogen. However, only a few forward genetic screens have been attempted, with low efficiency. Here, we designed a pipeline for efficient gene discovery in S. sclerotiorum (Fig. 1). First, ascospores were collected from apothecia of wild-type (WT) strain 1980 and were mutagenized by UV treatment. After testing different ascospore concentrations and UV exposure times (Supplementary Fig. S1), we settled on a UV exposure period of $15 \mathrm{~s}$ of irradiation, with 3,000 spores per plate inoculum. Under this UV dosage, $99 \%$ of WT ascospores are killed by UV irradiation. Through screening about 40,000 surviving spores, 32 mutants with visible morphological defects, i.e., discolored sclerotia or malformed or absent sclerotia, were identified (Supplementary Table S1). Upon transferring these morphologically distinguishable mutants from 96-well plates, we did not observe sectoring in their colonies on standard PDA (potato dextrose agar) plates, suggesting the homogeneity of the mutants. These mutants were further purified by at least three rounds of hyphal tip transfer to confirm that the mutant phenotypes were stable.

\section{Identification of mutants with obvious morphological phenotypes.}

Among these morphological mutants, three showed pigmentation defects during sclerotial formation. The WT strain formed black sclerotia (Fig. 2). However, the color of sclerotia is pinkish in pnk1 (pink 1) and pnk2 and brown in M98 (Fig. 2A). In addition, a large number of mutants did not form sclerotia (Fig. $2 \mathrm{~B}$ and $\mathrm{C}$ ). Although their ability to form sclerotia was abolished, every mutant exhibited slightly different mycelial morphology. M73, M77, L12, and R122 formed a hyphal ring at the edge of the plate where sclerotia normally form in the WT strain. WT mycelia grew flat on the surface of the media, while R10 produced extremely fluffy aerial mycelia covering up the entire plate. R7 and M30 produced small sclerotial primordia and white compact hyphal masses but could not develop further to form mature sclerotia (Fig. 2B). WT mycelia are hyaline. R1 and R213, however, had dark-colored mycelia (Fig. 2C). Viewed from both the front and back, mycelia of these two mutants seemed highly melanized. Furthermore, the remaining eight mutants exhibited altered sclerotial size or number (Fig. 2D). WT usually forms 25 to 30 sclerotia on a standard 90-mm PDA plate. However, M35 and L10 formed more than 100 smaller-sized sclerotia on each plate. The other six mutants produced fewer sclerotia. Detailed phenotypic analysis of all 32 mutants is summarized in Supplementary Table S1. Taken together, this pipeline is highly effective in identifying mutants with desired observable phenotypes. Hereafter, we used the two pink-colored mutants pnkl and pnk2 as a proof of concept for establishing an efficient gene discovery pipeline.

\section{pnk1 and pnk2 contain different mutations in the same gene.}

To identify the molecular lesions in the pnk mutants, pnkl was first cultured for genomic DNA extraction and its full genome was sequenced by NGS. M98, which forms brownish sclerotia, was also subjected to NGS. The genomic sequence of M98 was used to identify background mutations that occur in both pnk and M98 to further reduce the number of candidate mutations. An NGS sequence analysis pipeline was modified for $S$. sclerotiorum from our previously used mapping-by-sequencing protocol for Arabidopsis (Huang et al. 2019). Using the pipeline guided by GATK best practices, a total 593 SNPs (single nucleotide polymorphisms) and 846 INDELs (insertions and deletions) were captured in pnkl, including mutations in exonic, intronic, upstream, downstream, and intergenic regions. After comparison with the M98 sequence, 103 SNPs and 166 INDELs were found to be unique to pnk1. Among them, only 21 SNPs and eight INDELs were in exons. We excluded synonymous, intronic, or intergenic mutations as candidates, since they are less likely to cause significant consequences to the encoded proteins. Since the mapped reads for 18 SNPs and all INDELs were not homogeneous, they were likely from mismatches of short reads to the reference genome. Therefore, these mutations were eliminated. Three remaining SNP mutations that led to nonsynonymous changes in the pnkl genome became the prime candidate mutations and were kept for further analysis (Fig. 3A). Among these three SNPs, one resulted in a premature stop codon while the other two caused single amino acid changes.

To determine which of the three mutations is responsible for yielding the pink sclerotia phenotype, the protein sequences of these genes were analyzed to examine whether these genes or their orthologs in other organisms have been previously studied with reported functions. From this literature search, one gene (sscle_12g091490) stood out, which encodes the ortholog of a transcriptional factor (TF), BcSMR1 (Sclerotial melanogenesis regulatory gene 1) from Botrytis cinerea. A mutation in BcSMRI led to orange-colored sclerotia in $B$. cinerea, which normally forms black sclerotia (Zhou et al. 2017). We therefore renamed this ortholog of BcSMR1 in S. sclerotiorum as SsSMR1, and it became our top candidate for pnkl. SsSMR1 and BcSMR1 share 93 and $87 \%$ amino acid similarity and identity, respectively.

Due to the similar color of sclerotia between pnk1 and pnk2, Sanger sequencing was performed on SsSMRl with DNA from pnk2. A different mutation also causing a premature stop codon in SsSMR1 was identified in pnk2 (Fig. 3B). This suggests that mutations of SSSMRI in both pnk mutants are likely responsible for causing the same pink sclerotia phenotype. SsSMRI contains five exons and four introns, with the mutated nucleotides in pnkl and pnk2 both occurring in exon 5 (Fig. 3C). As these truncation mutations in SSSMRl likely cause loss-of-function of the protein, pnk1 and pnk2 were renamed Sssmrl-1 and Sssmrl2 , respectively.

Protein BLAST and motifs analysis by MEME Suite (Bailey et al. 2009) using the full-length 937-aa SsSMR1 supported its role as a TF. SsSMR1 has predicted DNA-binding domains at its $\mathrm{N}$-terminus, with two $\mathrm{Cys}_{2} \mathrm{His}_{2}$-zinc finger motifs and one 
$\mathrm{Zn}_{2} \mathrm{Cys}_{6}$-binuclear motif. In addition, SsSMR1 contains a fungalspecific, 298 aa long TF domain and a nuclear localization sequence (NLS) at its C-terminus (Supplementary Fig. S2). The Y706* and Q749* transitions result in the formation of a truncated TF domain in Sssmrl-2 and the loss of the NLS in both Sssmr1-2 and Sssmr1-1. Sequence alignment of orthologs of SsSMR1 and BcSMR1 in other representative Ascomycota fungi Colletotrichum lagenarium, Magnaporthe oryzae, and Bipolaris maydis revealed that the two mutated amino acids are not conserved (Fig. 3D), however, these TF-related motifs are highly conserved (Supplementary Fig. S2). As Botrytis and Sclerotinia are closely related, these results suggest that SsSMR1 is likely a transcription factor with similar functions.

Further phylogenetic analysis showed that orthologs of SsSMR1 are absent in prokaryotes, plants, and animals. It is only found in several classes of division Ascomycota and can be found nowhere else in the other lineages, including yeast (Supplementary Fig. S3). Meanwhile, SMR1 sequences from S. sclerotiorum and B. cinerea form a monophyletic Sclerotiniaceae group with a bootstrap of $100 \%$. SsSMR1 is highly homologous to the orthologs from order Helotiales within the clade of Leotiomycetes fungi and is conserved in species of the classes Sordariomycetes and Dothideomycetes.

\section{Generate ascospores}
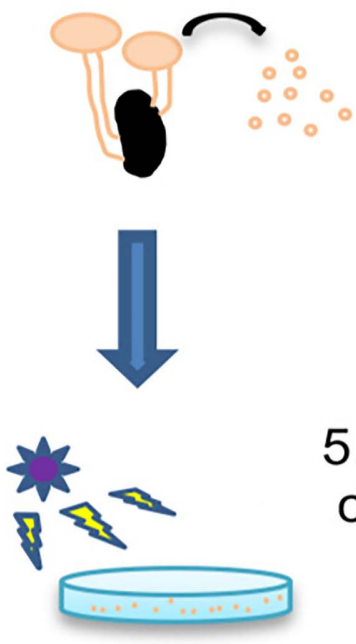

\section{UV mutagenesis of ascospores}

\section{Mutant purification, characterization and DNA extraction}
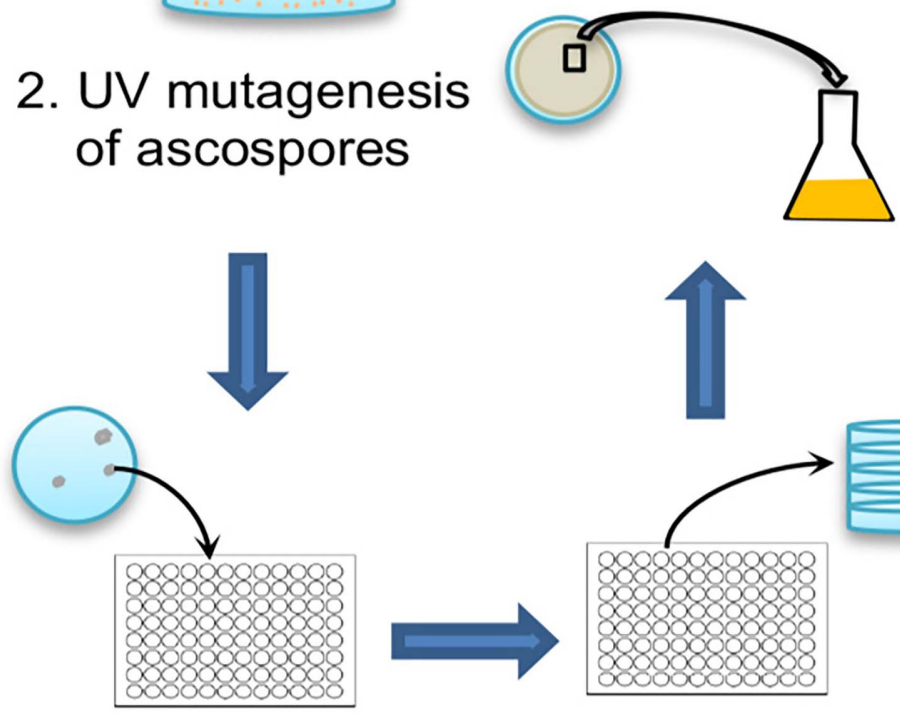

3. Individually transfer colonies to 96-well plates

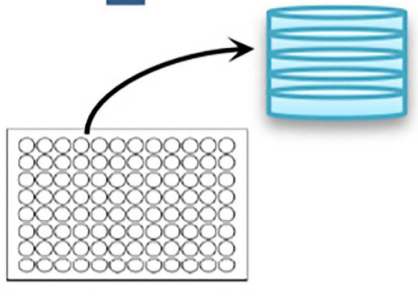

\section{Phenotyping and select} mutants
6. NGS

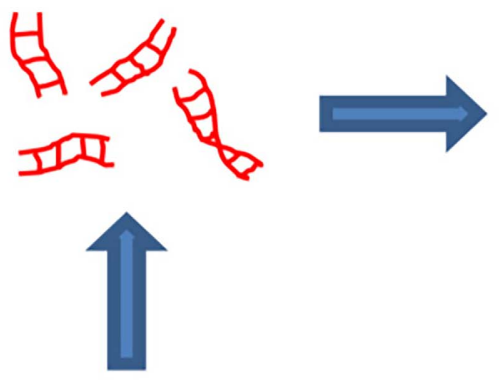

7. Identify

candidate genes

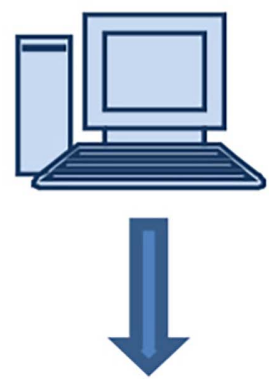

8. Complementation or gene knock-out for confirmation

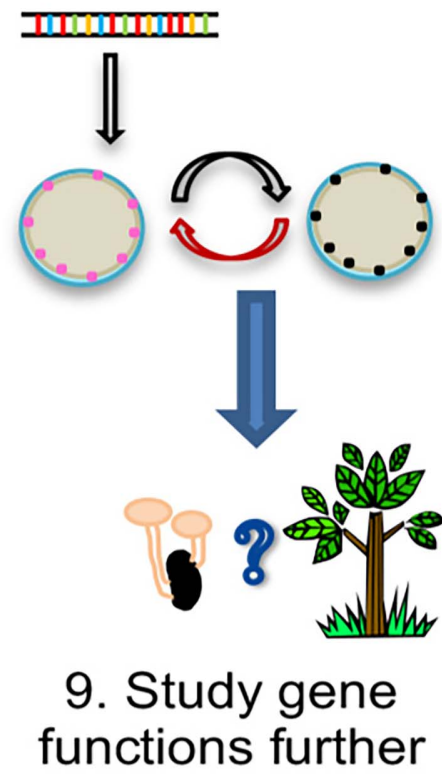

Fig. 1. A pipeline designed for forward genetic analysis of Sclerotinia sclerotiorum. Step 1: The haploid ascospores of the wild-type (WT) strain S. sclerotiorum 1980 are collected from apothecia that were germinated carpogenically from sclerotia. Step 2: The ascospores are subjected to UV mutagenesis. Step 3: After UV treatment, the germinating colonies from ascospores are transferred individually into 96-well potato dextrose agar (PDA) plates and are incubated for 8 to 10 days to observe for phenotypes. Step 4: The mutants showing target phenotypes (nonsclerotial formation or discolored sclerotia) are transferred onto a fresh PDA plate (90 mm diameter) for confirmation. Step 5: Confirmed mutants are subjected to DNA extraction. Step 6: Genomic DNA of the mutants are sequenced by nextgeneration sequencing (NGS). Step 7: After NGS data analysis, candidates can be identified for each mutant. Step 8: Target gene complementation or knockout approaches can be used to identify the exact gene responsible for the observed mutant phenotype. Step 9: The biochemical mechanism of the related proteins can be studied further by various means depending on the nature of the protein. 
Targeted gene disruption and transgene complementation confirmed that SsSMRI

is the responsible gene for Sssmr1 phenotype.

To confirm that mutations in SSSMRl are responsible for the sclerotial pigmentation deficiency in Sssmrl-1 and Sssmr1-2, targeted gene replacement by homologous recombination was performed in WT background (Fig. 4A). The Sssmrl transformants were selected on PDA with $50 \mu \mathrm{g}$ of hygromycin per milliliter, and two independent positive transformants, Sssmrl-3 and Sssmr1-4, were subjected to three rounds of hyphal tip transfer and protoplast purification to obtain pure knockout mutants. The pure disruption of SSSMRI in these knock-out transformants was verified by PCR (Fig. 4B). A 965-bp SSSMRI single band was present in WT but completely absent in the two transformants. The presence of selection marker gene hygromycin phosphotransferase $(h p h)$ in the transformants was further confirmed by the amplification of a 799-bp product. In addition, the upstream 1,567-bp and downstream 1,413-bp amplicons suggest that the gene replacement occurred as designed and did not disrupt other genes (Fig. 4B). After culturing on PDA medium for 14 days, pink sclerotia formed on the plate edges for both Sssmr1-3 and Sssmrl-4 (Fig. 4C), resembling the phenotypes of Sssmr1-1 and Sssmrl-2. Therefore, knocking out SSSMRl leads to pink sclerotia.
Furthermore, when WT SSSMRI was cloned and transformed into Sssmr1-1, two complementing stains SsSMR1-C1 and SsSMR1-C2 (Fig. 4C) were recovered, which formed black sclerotia. Taken together, these data suggest that SSSMRI is the gene responsible for the pink sclerotia phenotype observed in the Sssmrl mutants isolated from our screen. It, indeed, has an important role in sclerotial melanization. Loss of SSSMRl blocks melanin biosynthesis, leading to pink sclerotia.

\section{Sclerotia melanization does not contribute}

to virulence and compound appressoria formation, but it does contribute to sclerotial surface structure and apothecia formation.

We used a similar pipeline to analyze M98. Interestingly, a nonsynonymous change in the fourth exon of another melanin biosynthetic gene, SsPKS12 (sscle_12g091500), which is adjacent to SSSMRl in the genome, was identified in M98 (Fig. 5A). This mutation results in an I584F change. Consistent with the phenotype of Sspks12 observed by Wang and Rollins (2021), M98 formed brown sclerotia (Fig. 2A). These data indicate that the observed mutation in SsPKS12 is most likely responsible for the M98 mutant phenotype.
A

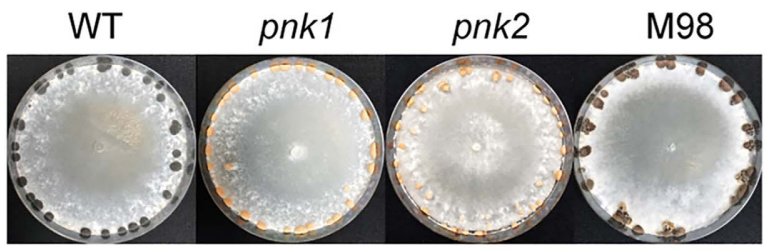

B
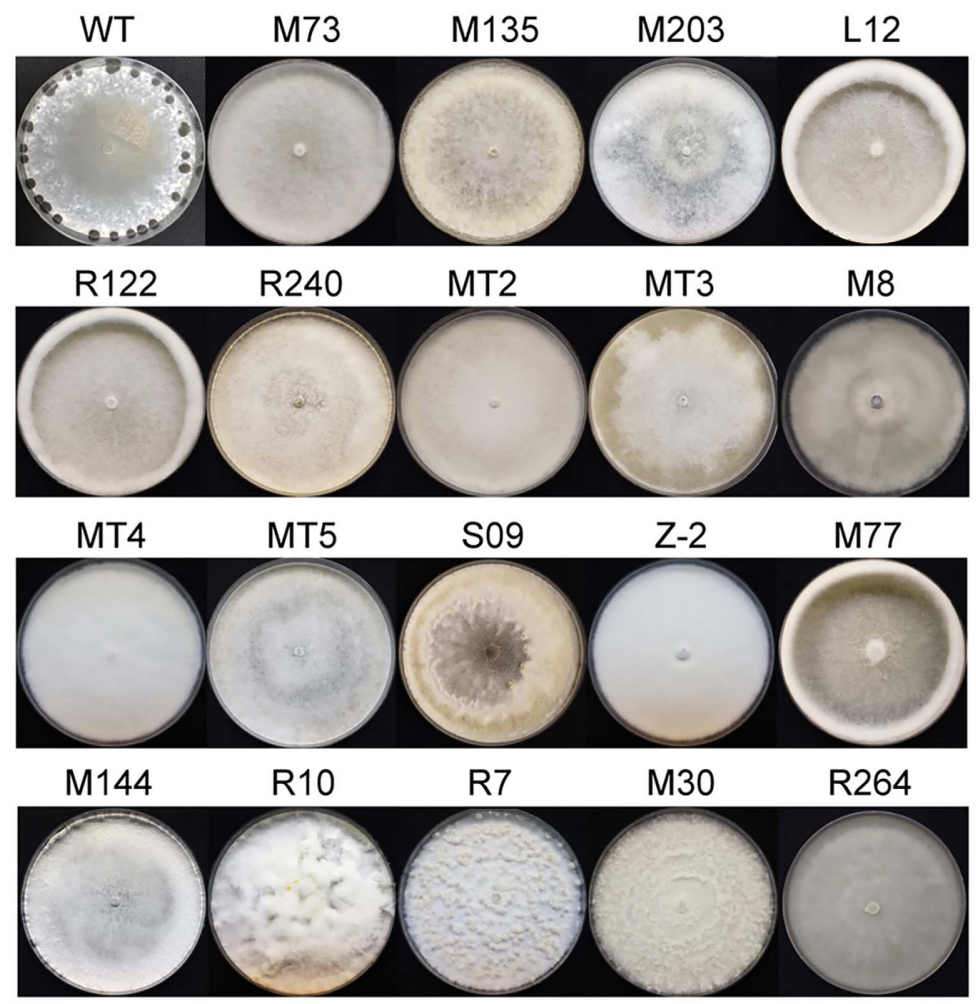

C

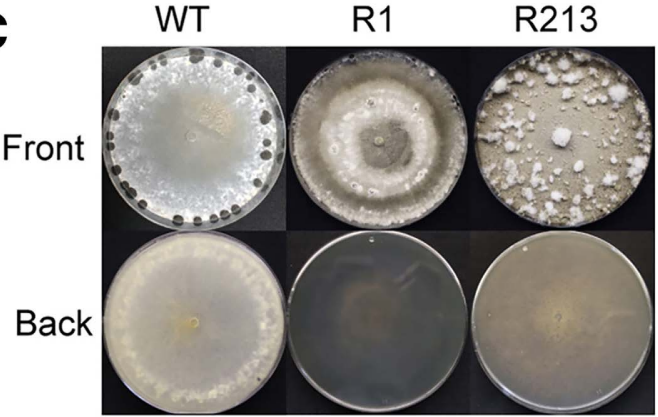

D

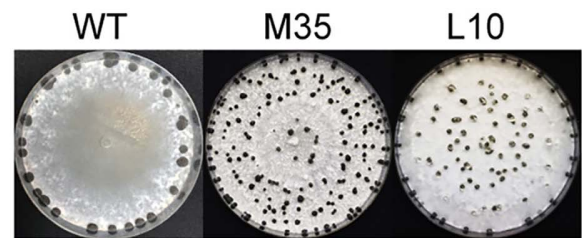

L11

M131

M133

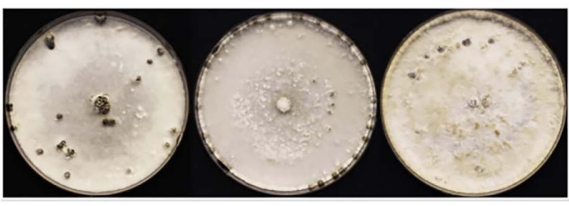

MT1

M25

M42

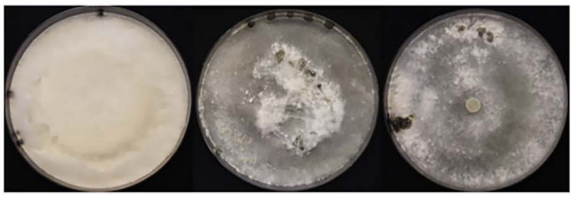

Fig. 2. Colony morphology of mutants with sclerotial defects. A, Wild-type strain Sclerotinia sclerotiorum 1980 (WT) and mutants with discolored sclerotia. B, Mutants not forming sclerotia. C, Mutants with dark-colored mycelia. Photos were taken from both front and back of the plates. D, Mutants with altered sclerotial size or number. The WT strain and all mutants were cultured on potato dextrose agar plates for 2 weeks before the pictures were taken. 
With the availability of these melanization-deficient mutants, we tested the biological roles of sclerotial melanization in Sclerotinia biology. Sssmr1-1, Sssmrl-2, Sssmrl-3, Sssmrl-4, and M98 mutants showed no significant differences from WT in growth rate (Fig. 5B), demonstrating that sclerotia melanization does not affect vegetative growth. Unlike in other fungi such as M. oryzae, in which melanin contributes greatly to pathogenicity (Howard and Ferrari 1989), sclerotial melanin does not seem to be required for establishing infection in S. sclerotiorum, as the Sssmrl mutants and M98 are not compromised in their pathogenicity and virulence, compared with the oahl mutant, which is an oxalate-deficient mutant exhibiting significantly attenuated virulence (Liang et al. 2015) (Fig. 5C). This is consistent with previous reports that disruption of Sspks12 and Sspks13, two enzymes encoding genes required for melanin accumulation in sclerotia and apothecia and in compound appressoria ( $\mathrm{Li}$ et al. 2018; Wang and Rollins 2021), respectively, did not affect virulence. Since Sssmr1-1, Sssmr1-2, Sssmrl-3, and Sssmrl-4 behaved similarly, we chose Sssmrl-1 as representative to perform assays hereafter.

When WT, Sssmr1-1, and M98 were cultured on glass slides for the observation of compound appressoria development and pigmentation, we did not observe any defects (Fig. 5D); they produced compound appressoria with wild-type pigmentation and morphology. Thus, SsSMR1 and SsPKS12 do not seem to contribute to melanization of appressoria. Sclerotial melanization seems independent of melanization of appressoria, which is consistent with the previous report (Wang and Rollins 2021).

Next, the surface structure of these mutants was observed with a dissecting microscope. Compared with WT sclerotia, which are rough surfaced with many bumpy structures, sclerotia of M98 show reduced bumpiness, while the Sssmrl-1 mutant is smooth-surfaced (Fig. 5E), indicating that SsSMR1 and SsPKS12 likely contribute to the surface structure of the sclerotia. When we produced apothecia, Sssmrl-1 and M98 show significantly reduced ability to produce apothecia when compared with WT (Fig. 5F). And the apothecium produced from M98 was albino, which is consistent with the apothecial pigmentation defect in Sspks12 mutants (Wang and Rollins 2021).

\section{Sclerotial melanization is required for survival of sclerotia under physical and chemical stresses.}

As highly melanized sclerotia were hypothesized to be responsible for resisting physical, chemical, and biological stresses (Bolton et al. 2006), we next tested whether sclerotia of the melanization deficiency mutants can withstand toxic bleach treatment or UV irradiation and germinate properly. After $60 \mathrm{~min}$ of bleach treatment or $20 \mathrm{~min}$ of UV irradiation, the
A

\begin{tabular}{cccc}
\hline $\begin{array}{c}\text { Chrom } \\
\text { osome }\end{array}$ & $\begin{array}{c}\text { Nucleotide } \\
\text { change }\end{array}$ & $\begin{array}{c}\text { AA } \\
\text { change }\end{array}$ & Reference gene \\
\hline 4 & G4376C & S1459T & sscle_04g033050 \\
6 & A685C & K229Q & sscle_06g050010 \\
12 & C2245T & Q749* & sscle_12g091490 \\
\hline
\end{tabular}

B

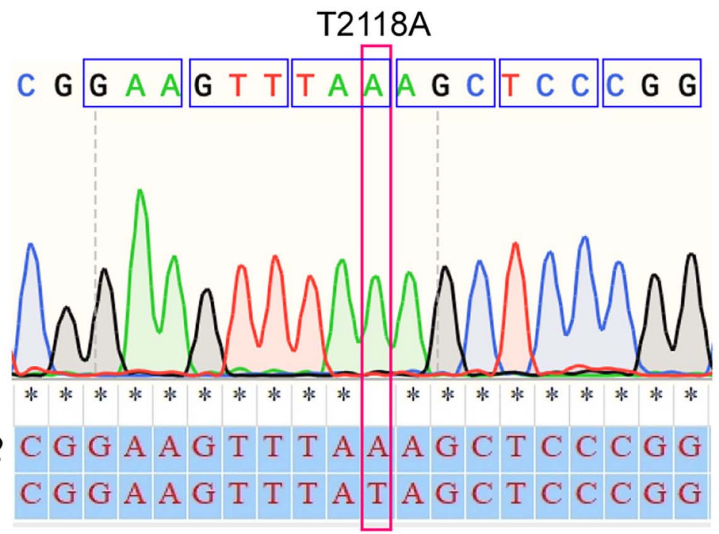

C

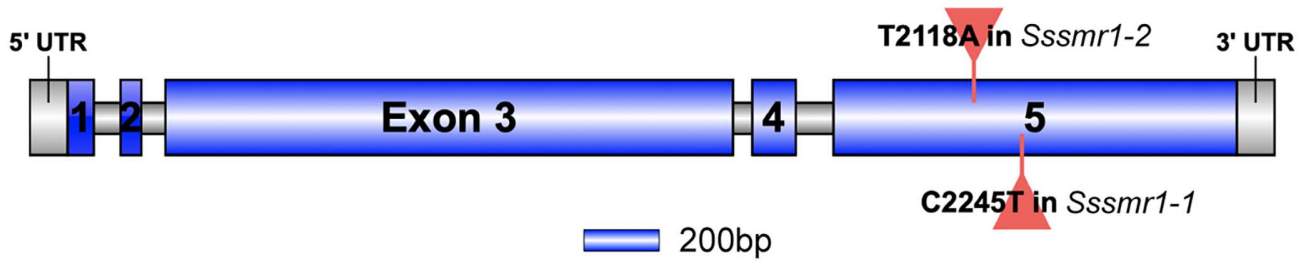

D

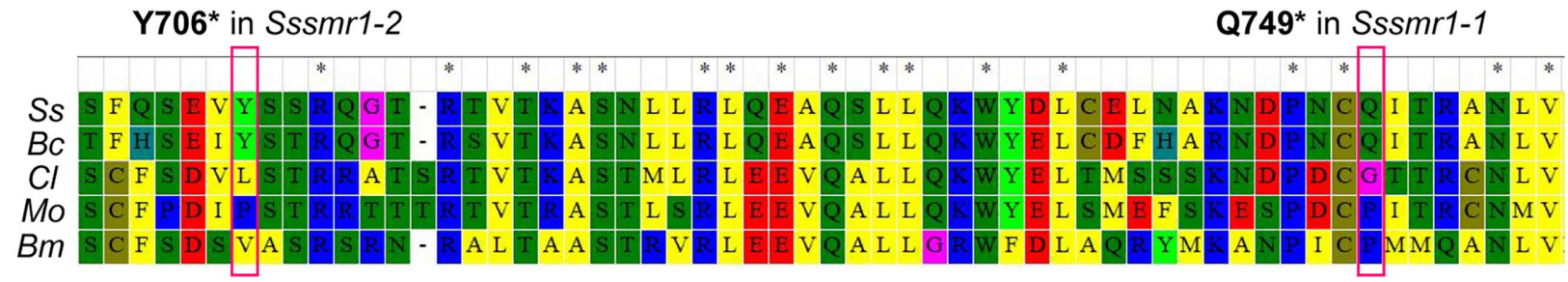

Fig. 3. Identification of the molecular lesions of the two pink (pnk) mutants. A, Candidate mutations of $P N K 1$ after next-generation sequencing data analysis. The most likely gene, SsSMR1 (sscle_12g091490), is highlighted in red. B, Sanger sequencing chromatograph of SsSMR1 in pnk2. The nucleotide altered is marked in a red box. The nucleotide triplets coding amino acids are labeled in blue boxes. The asterisks represent nucleotides that remain unchanged. C, Gene structure of SsSMR1. The diagram was drawn using Illustrator for Biological Sequencing (Liu et al. 2015), including exons, introns, $5^{\prime}$ untranslated region (UTR), and 3' UTR. The mutation sites in Sssmr1-1 and Sssmrl-2 are labeled with arrowheads. The scale bar is shown at the bottom. D, Protein sequence alignment of the region containing the two Sssmr1 mutations with orthologs of SsSMR1 in different fungal species. The amino acids changed in Sssmr1-1 and Sssmr1-2 are highlighted in red. The asterisks represent the conserved amino acids. 
myceliogenic germination rate declined significantly by 67 and $51 \%$ for Sssmrl-1 sclerotia and 37 and $21 \%$ for M98 sclerotia, respectively, as compared with WT (Fig. 5G and H). Taken together, melanization protects sclerotia from abiotic environmental stresses such as UV or toxic chemicals.

\section{The melanin biosynthesis pathway is regulated by SsSMR1.}

To analyze the role of the SsSMR1 TF in melanin biosynthesis in more detail, we carried out RNA sequencing (RNA-seq) analysis. As melanin biosynthesis occurs as the fungus reaches maturity, young (4-day-old) and old mycelia (9-day old) potato dextrose broth (PDB)-grown WT and Sssmrl-1 were collected in triplicates for analysis (Fig. 6A). Distance matrix analysis of the samples confirmed that there is a high level of transcriptome similarity between biological replicates (Fig. 6B). Principal component analysis revealed that PC1, likely related to age, and PC2, likely related to genotype, explain 87 and $10 \%$ of the expression variance between samples (Fig. 6C). Agreeing with the morphological differentiation pattern, the transcriptomes of WT and Sssmr1-1 are quite similar in young hyphae but differ substantially in older hyphae going through sclerotial melanization.

To determine which specific genes are regulated by SsSMR1, differentially expressed genes (DEGs) were identified using thresholds of $\log 2$ (fold-change) $>0.8$ or $<-0.8$ and padj $<0.05$ (Supplementary Table S2). Relatively few genes (282) were significantly affected in Sssmrl-1 young hyphae compared with WT (Fig. 6D to E). Upon maturation, 1,057 and 1,179 genes are up- and down-regulated, respectively, in WT. This constitutes $20 \%$ of the 11,130 coding genes in $S$. sclerotiorum. The genes upregulated in old compared with young WT hyphae are enriched in gene ontology (GO) terms related to secondary metabolite biosynthesis, copper ion transport, and melanin biosynthesis, consistent with the involvement of these processes in melanization during sclerotia development (Fig. 7A). Compared with WT, fewer genes were downregulated and more genes were up-regulated in Sssmrl-1 upon maturation. Specifically, 218 and 385 genes were found to be significantly down- and up-regulated, respectively in old Ssmr1-1 mycelia compared with old WT mycelia. These 218 downregulated genes were enriched in GO terms related to melanin processes, further supporting the involvement of SsSMR1 in melanin biosynthesis (Fig. 7B).

Previous studies in $B$. cinerea and other fungi revealed that SMR1 regulates several genes directly involved in melanin biosynthesis (Schumacher 2016). From the literature, we deduced a hypothetical melanin biosynthesis pathway in S. sclerotiorum (Fig. 7C). Polyketide synthase (PKS) enzymes PKS12/13 along with YGH1 ("Yellowish-green" hydrolase 1) generate T4HN (1,3,6,8-tetrahydroxynaphthalene). This precursor is converted to T3HN (1,3,8-trihydroxynaphthalene) and then further to DHN (1,8-dihydroxynaphthalene) by sequential reactions catalyzed by BRN2 (T4HN reductase, also named THR1), BRN1 (T3HN reductase, also named THR2), and SCD1 (Scytalone dehydratase 1). Finally, a laccase, LAC1, converts DHN to DHN-melanin (Tsuji et al. 2001). Other proteins are also involved, such as the copper transporter CCC2 (Copper-transporting ATPase 2), which may import the copper necessary for laccases to function (Saitoh et al. 2010). In addition to these biosynthesis genes, various transcription factors, kinases, and the Velvet complex were also previously shown to play a role in melanin synthesis in other fungi (Schumacher et al. 2015; Yang et al. 2013).
A

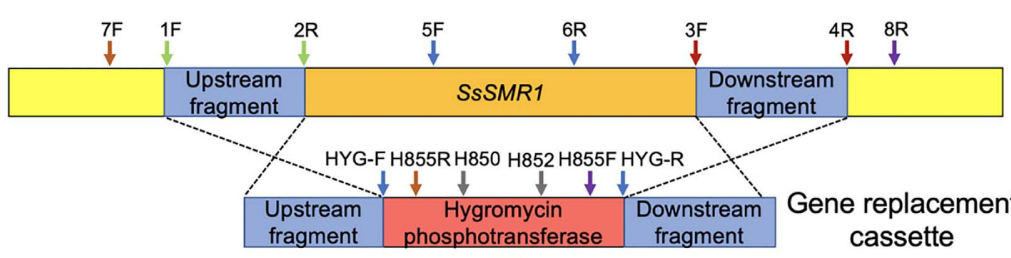

Homologous $\zeta$ recombination
B

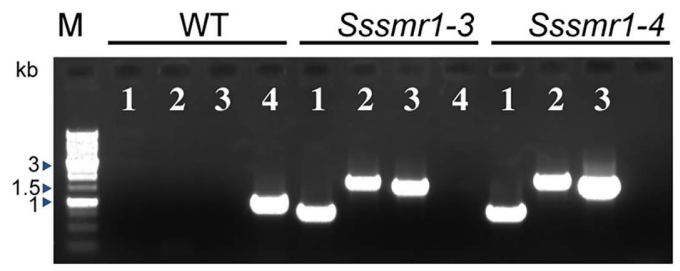

1. $\mathrm{H} 850+\mathrm{H} 852$ (799bp) 2. $7 \mathrm{~F}+\mathrm{H} 855 \mathrm{R}(1567 \mathrm{bp})$ 3. $H 855 F+8 R(1413 b p) 4.5 F+6 R(965 b p)$

\begin{tabular}{|l|l|c|c|c|}
\hline & $\begin{array}{c}\text { Upstream } \\
\text { fragment }\end{array}$ & $\begin{array}{c}\text { Hygromycin } \\
\text { phosphotransferase }\end{array}$ & $\begin{array}{c}\text { Downstream } \\
\text { fragment }\end{array}$ & \\
\hline
\end{tabular}

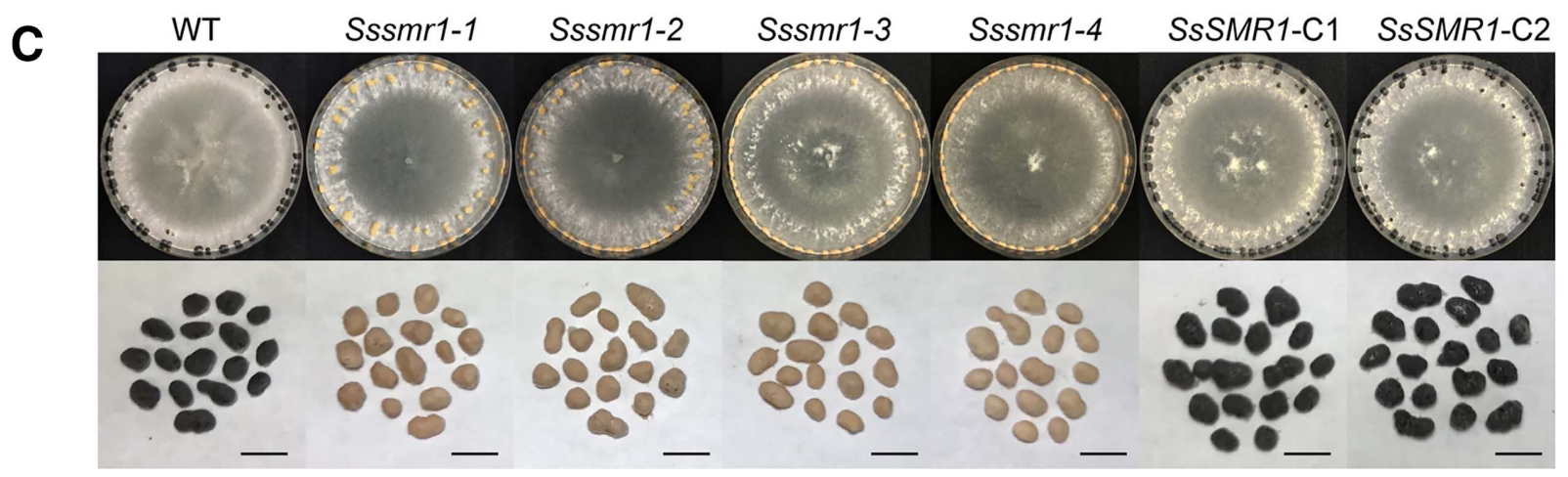

Fig. 4. Generation of Sssmr1 deletion mutants and complementation strains. A, The SsSMR1 locus and the gene replacement design. The SsSMR1 and hygromycin phosphotransferase genes $(h p h)$ are presented as orange and red rectangles, respectively. The primers labeled in the diagram were used to amplify the flanking sequences or for mutant screening. B, PCR verification of SsSMR1 disruption. Genomic DNA isolated from wild type (WT) and deletion mutants Sssmr1-3 and Sssmrl-4 were used as PCR templates. Four pairs of primers for checking the insertion of $h p h$, upstream and downstream of $h p h$ insertion and SsSMRl deletion, are listed and the sizes of amplified bands are shown in brackets. $\mathrm{M}=$ the lane for DNA marker. C, Colony morphology of WT, Sssmr1-1, Sssmr1-2, Sssmr1-3, Sssmr1-4, SsSMR1-C1, and SsSMR1-C2. All strains were cultured on potato dextrose agar plates for 2 weeks before the pictures were taken. Sclerotia collected from the plates are shown on the right. Scale bar $=5 \mathrm{~mm}$. 
We first investigated whether S. sclerotiorum homologs of these melanin biosynthesis-related genes are expressed in older hyphae and whether their expression depends on SsSMR1 (Fig. 7D; Supplementary Table S3). Strikingly, key enzymes
PKS12/13, YGH1, BRN1/2, and SCD1 are all up-regulated in older WT mycelium compared with young mycelia in WT $S$. sclerotiorum (Fig. 7D), supporting their roles in melanization. Interestingly, the expression levels of all these enzymes are

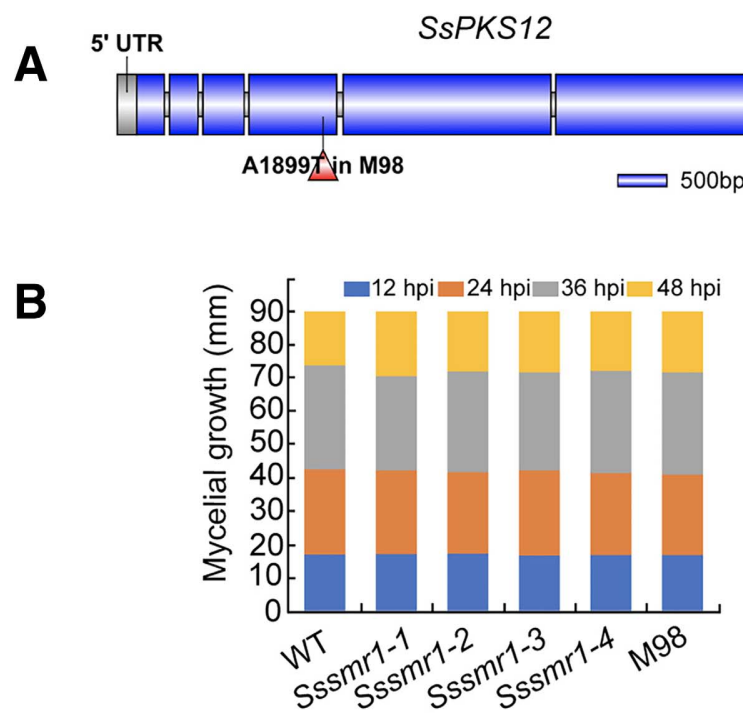

D

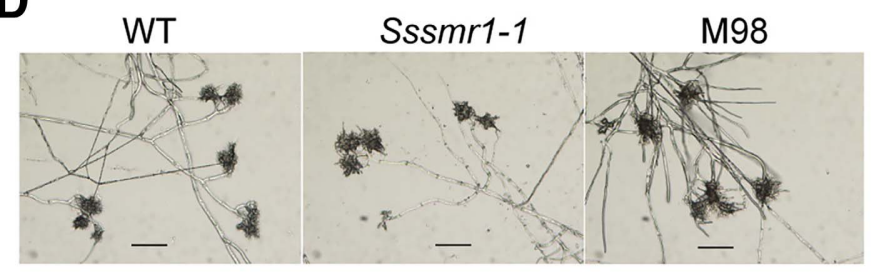

E

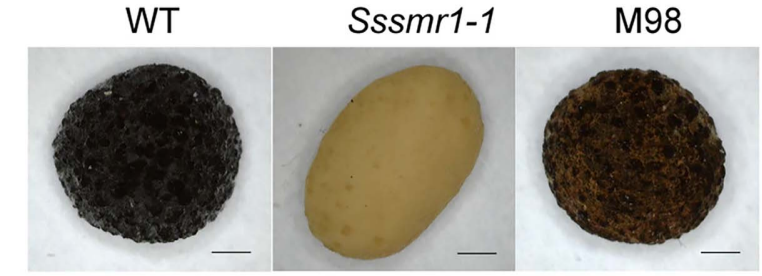

$\mathbf{F}$

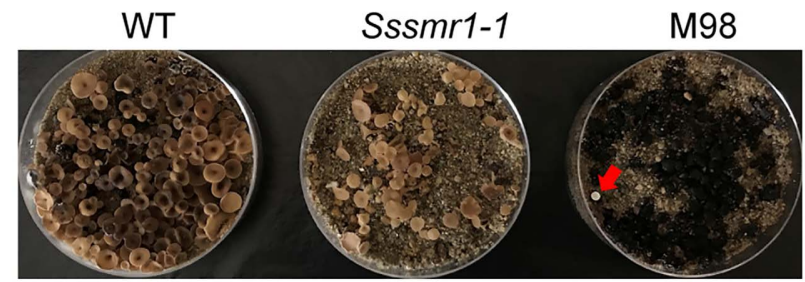

3' UTR
C

G

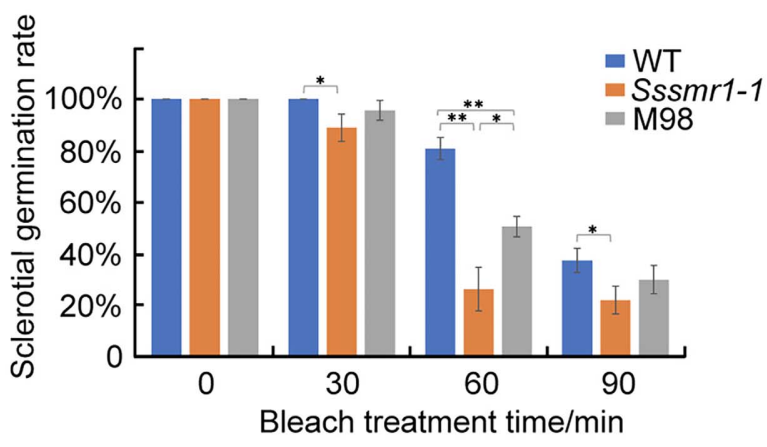

H

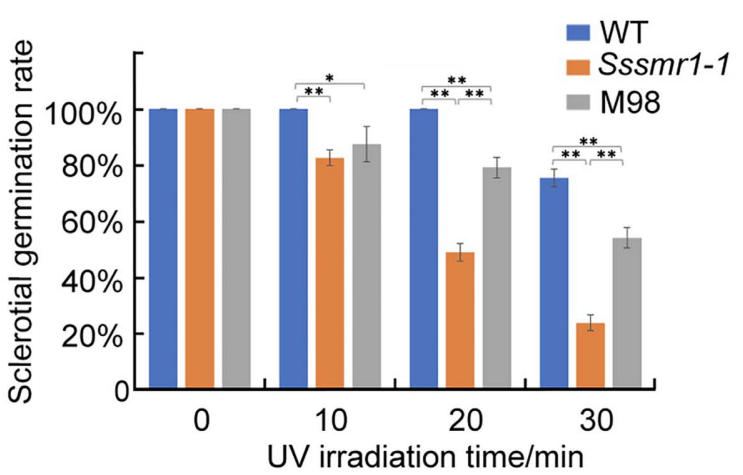

Fig. 5. Testing Sssmr1 and M98 mutants for defects in growth, virulence, and bleach or UV sensitivity. A, Gene structure of SsPKS12. The diagram was drawn using Illustrator for Biological Sequencing. Exons and introns are indicated in blue and gray boxes, respectively. The mutation site in M98 is labeled with an arrowhead. The scale bar is shown at the bottom. B, Mycelial growth of wild type (WT), Sssmr1-1, Sssmr1-2, Sssmr1-3, Sssmr1-4, and M98 over a period of $48 \mathrm{~h}$ with 12-h time intervals. C, Pathogenicity assay for WT, Sssmr1-1, Sssmr1-2, Sssmr1-3, Sssmr1-4, M98, and oah1 on detached leaves of Arabidopsis. Representative photos were taken at 24 and $48 \mathrm{~h}$ postinoculation, respectively. D, Compound appressoria formation assay. Potato dextrose agar plugs colonized with WT, Sssmr1-1, and M98 were transferred onto glass slides and were then photographed at 2 days postinoculation. The scale bar is $100 \mu \mathrm{m}$. E, Two-week-old sclerotia of WT, Sssmr1-1, and M98 were photographed by a dissecting microscope. Scale bar = $1 \mathrm{~mm}$. F, Apothecia formation assay. Sclerotia of WT, Sssmr1-1, and M98 were cultured in Petri dishes (90 mm diameter). The red arrowhead indicates the sole apothecium produced by sclerotia of M98. G and H, The sensitivity to $15 \%$ bleach treatment (C) and UV irradiation (D) of the WT, Sssmrl-1, and M98 strains. Asterisks indicate significance (two asterisks [**] indicate $P<0.01$ ) with one-way analysis of variance Tukey's post hoc test. No asterisk means no significance. 
significantly reduced in the older mycelia of Sssmr1-1, suggesting that SsSMR1 regulates these key melanin biosynthetic enzymes. SsSMR1 itself is also strongly up-regulated in older mycelia, indicating it is regulated during development. The expression level of SSSMRI is not significantly altered in the Sssmrl mutant, suggesting that the point mutation did not disrupt its transcription but, instead, affected its protein function. These data also suggest that SsSMR1 does not have a feedback role on its own transcription.

In contrast, the expression of putative upstream kinases, the Velvet complex, and other melanin-related TFs are not significantly affected in young or old mutant hyphae, suggesting that
A
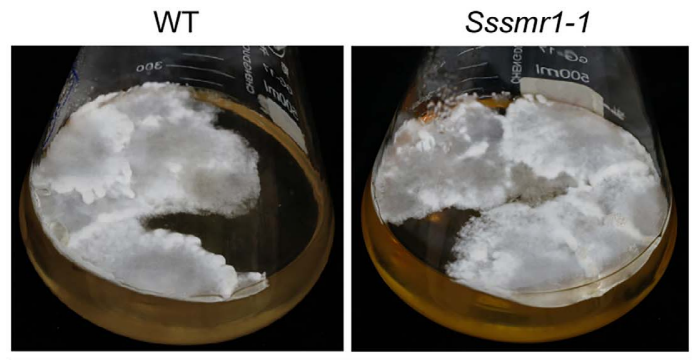

Young mycelia (4-day old)

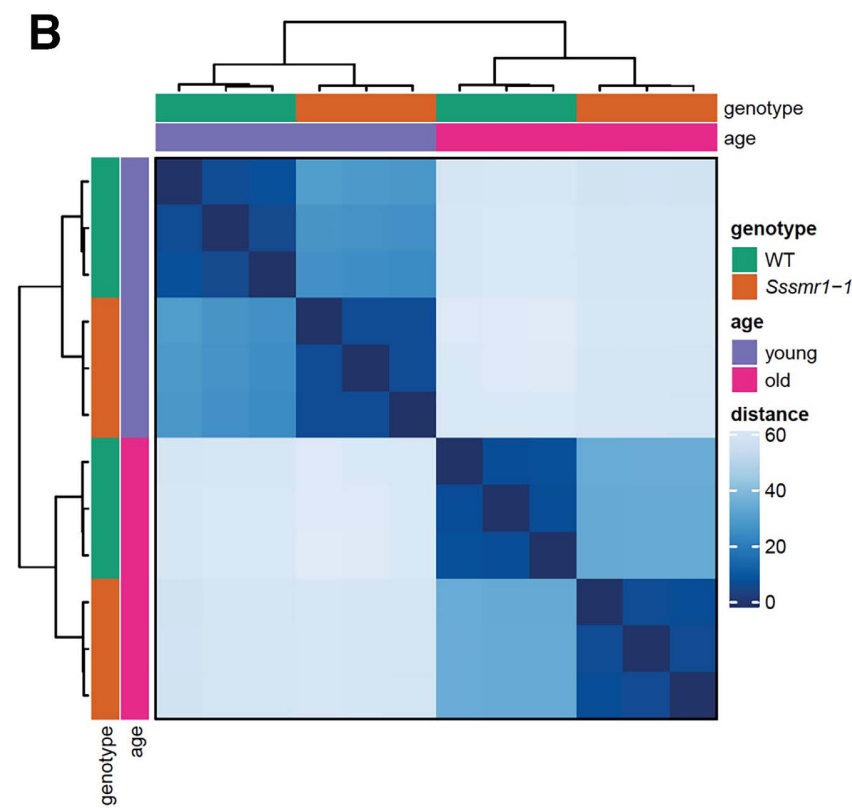

D

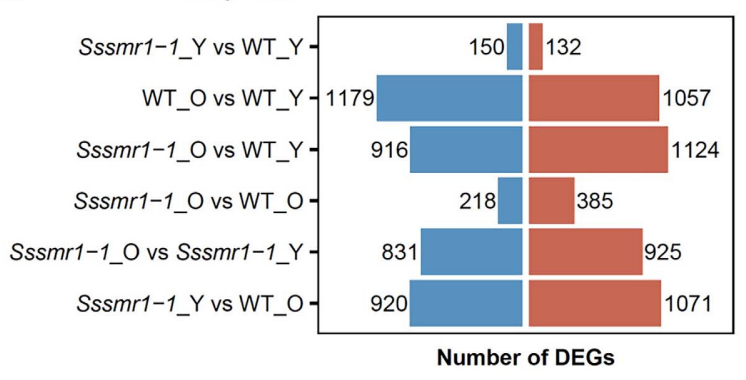

Relative expression: $\square$ Down $\square$ Up
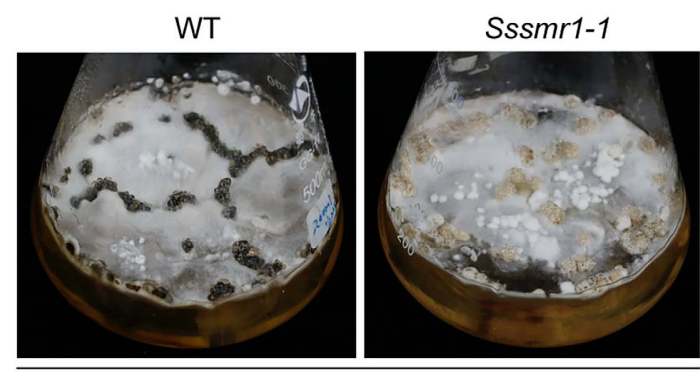

Old mycelia (9-day old)
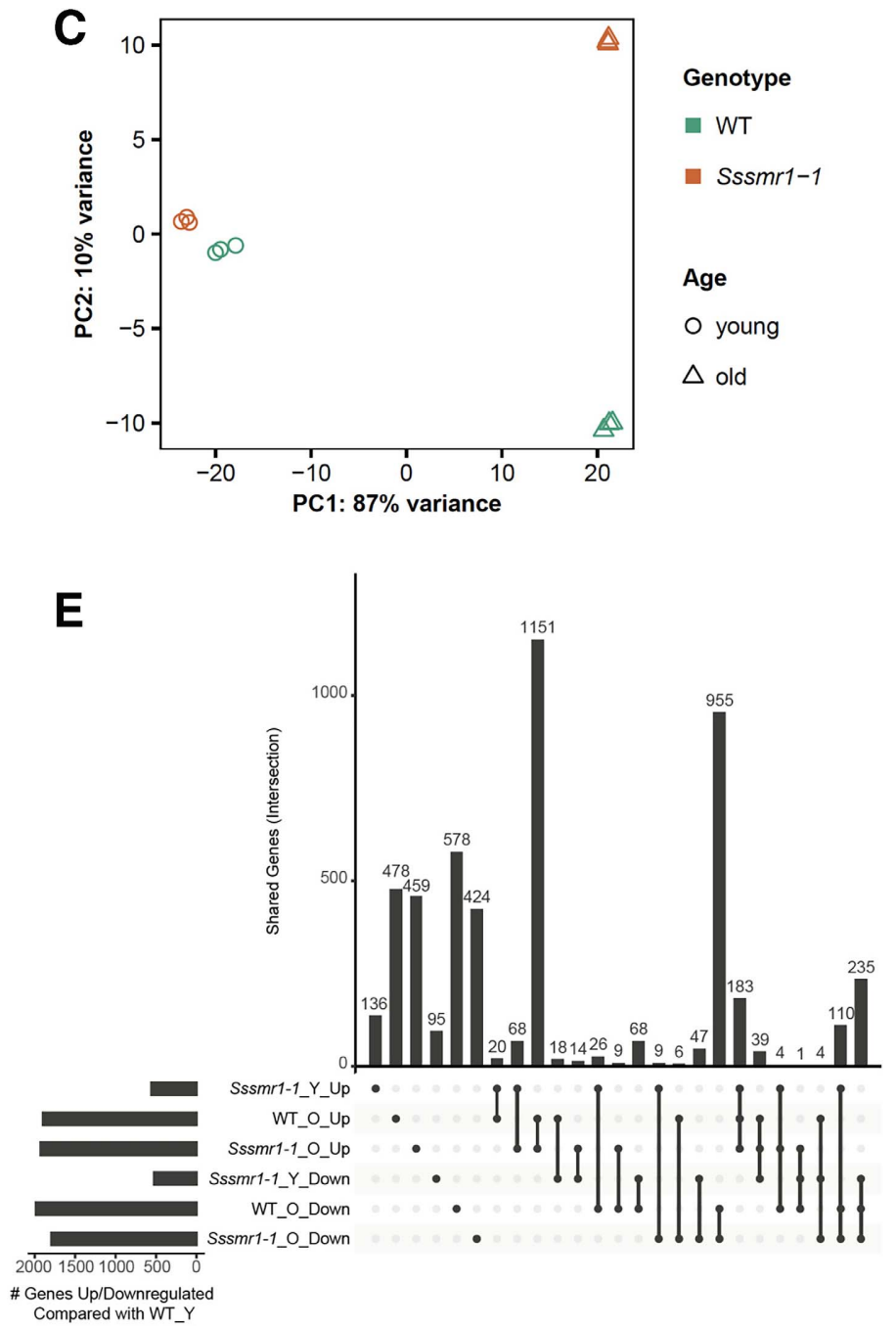

Fig. 6. RNA sequencing (RNA-seq) analysis of wild type (WT) and Sssmr1-1. A, Young and old mycelia were harvested from WT and Sssmr1-1 potato dextrose broth cultures at 4 and 9 days postinoculation, respectively. Photos were taken just before sample collection. B, Distance matrix between different RNA-seq samples. Heatmap showing Euclidean distance between different samples with values represented by a blue color scale. Hierarchical clustering of samples is shown by the top and left dendrograms. Different genotype and age groupings are denoted by colored bars on the top and left of the heatmap. C, Principal component analysis of gene expression in young and old WT and Sssmrl-1 hyphae. First two principal components are shown. Age is represented by shape, and genotype is represented by color. D, Comparisons of significant differentially expressed genes (DEGs) between different ages and genotypes. Numbers represent DEG count for respective categories. $\mathrm{Y}=$ young mycelia, $\mathrm{O}=\mathrm{old}_{\mathrm{m}} \mathrm{mcelia}$. Thresholds used were $\log _{2} \mathrm{FC}>$ 0.8 or $<-0.8$ and padj $<0.05$. E, Comparisons of significant differentially expressed genes between different ages and genotypes as compared with young WT mycelium. Upset plot showing DEGs shared between different groups. Vertical bars and corresponding value represent the distinct set between sets labeled with gray dots. $\mathrm{Y}=$ young mycelia, $\mathrm{O}=$ old mycelia. 
A
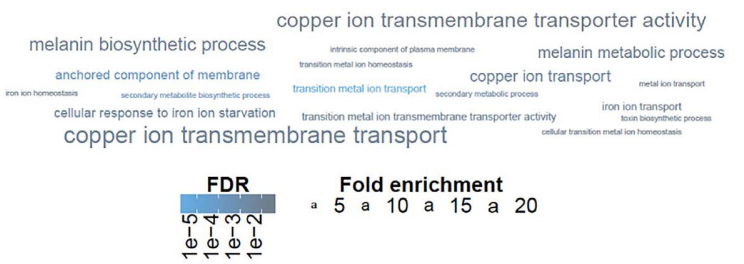

B

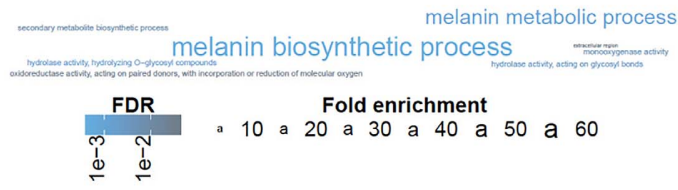

D

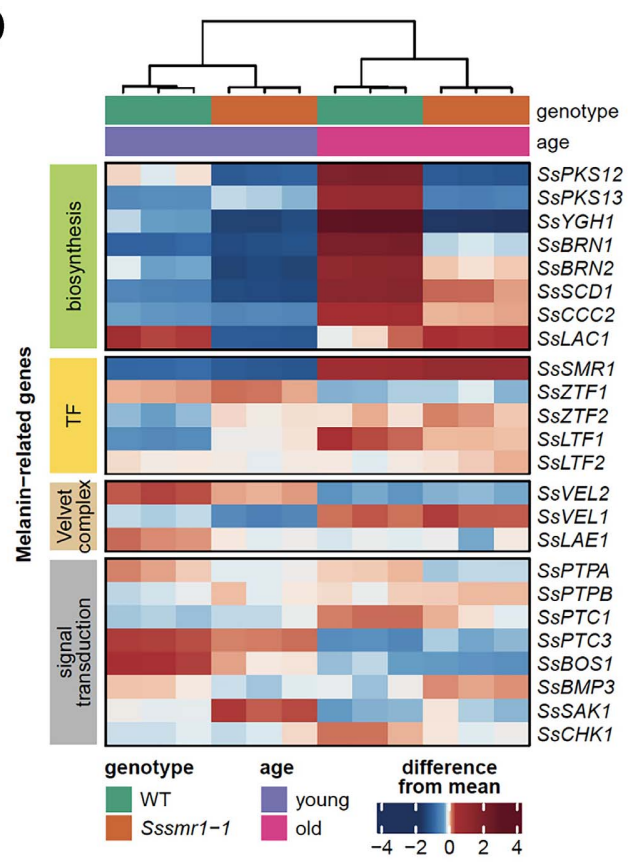

G Potential binding motif of SsSMR1

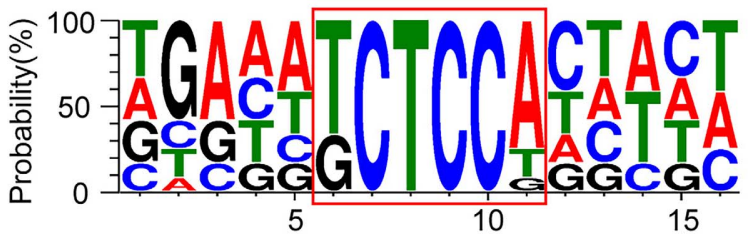

C

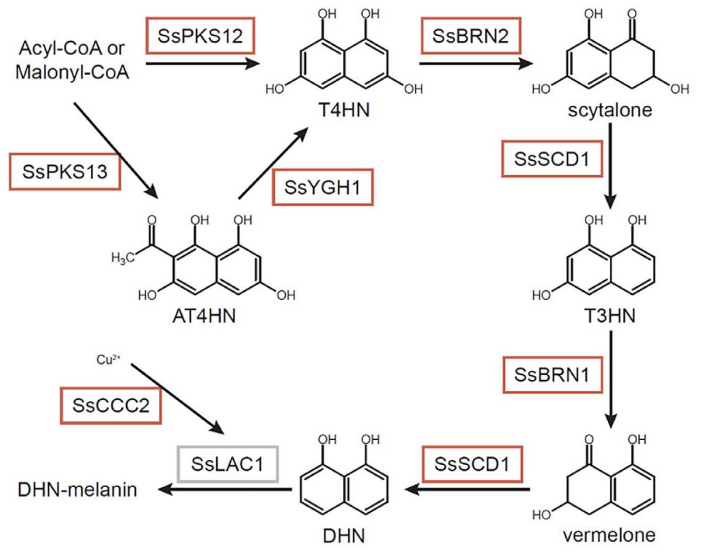

E

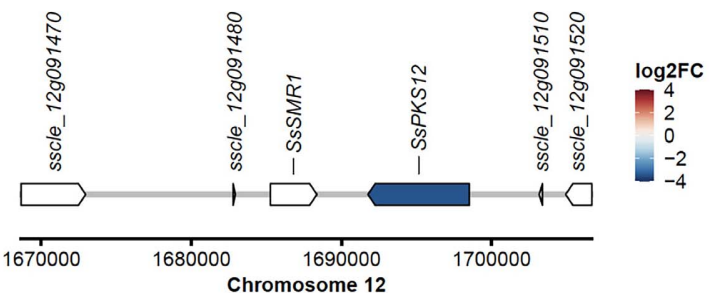

F

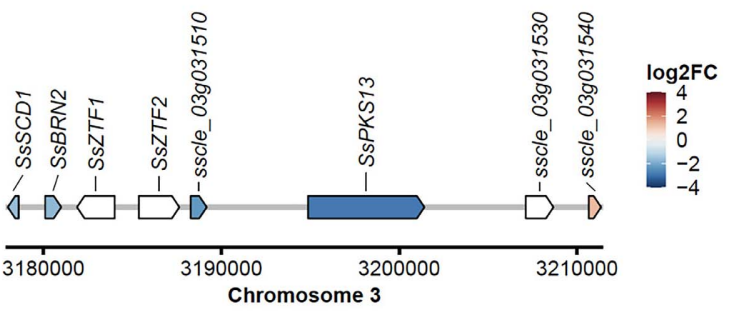

H

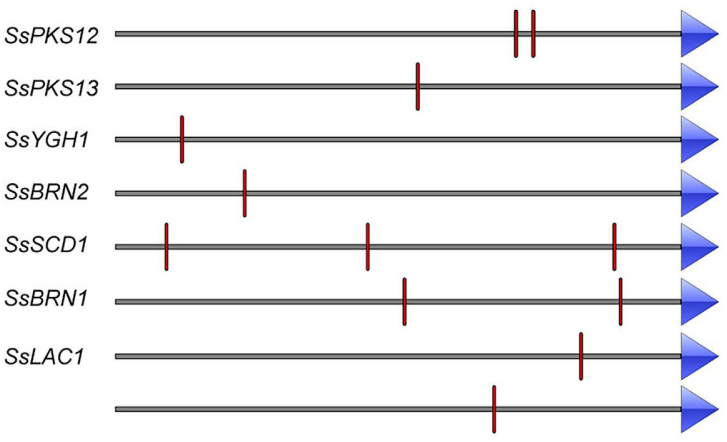

Fig. 7. SsSMR1 regulates the transcription of genes encoding melanin biosynthesis enzymes. A, Word cloud of gene ontology (GO) terms enriched in the gene set upregulated in old wild-type (WT) hyphae compared with young WT hyphae. Font size is proportional to fold enrichment (test ratio/reference ratio), and significance is represented by color. B, Word cloud of GO terms enriched in the gene set significantly downregulated in old Sssmrl-1 hyphae compared with old WT hyphae. Font size is proportional to fold enrichment (test ratio/reference ratio), and significance is represented by color. C, Proposed Sclerotinia melanin biosynthesis pathway deduced from homologous genes in other fungi. Enzymes with red borders are regulated by SsSMR1. Gene codes can be found in Supplementary Table S3. D, Expression profile comparisons of Sclerotinia sclerotiorum homologs of known melanin biosynthesis-related genes. Heatmap showing mean-centered, variance-stabilized transformation of transcript counts with values represented by a diverging blue and red color scale. Names of S. sclerotiorum homolog genes are listed on the right, grouped by the type of regulator (denoted on left). Hierarchical clustering of samples was completed and is represented by the dendrogram above the heatmap. Different genotype and age groupings are denoted by colored bars on top of the heatmap. E, Gene diagram showing the SsPKS12 and SsSMRI locus on chromosome 12. Genes found to be differentially regulated (threshold of $\log _{2} \mathrm{FC}>0.8$ or $<-0.8$, padj $<$ 0.05 ) in old SSSMR1 hyphae compared with old WT hyphae are colored. F, Gene diagram showing the SsPKS13 locus on chromosome 3. Genes found to be differentially regulated (threshold of $\log _{2} \mathrm{FC}>0.8$ or $<-0.8$, padj $<0.05$ ) in old SsSMRI hyphae compared with old WT hyphae are colored. G, Conserved binding motif identified in the promoter regions of melanin biosynthesis pathway genes. The $x$ axis shows nucleotide positions, the $y$ axis indicates sequence conservation at each position. The height of symbols represents the probability of each nucleotide occurring at this position. The diagram was drawn by WebLogo (Crooks et al. 2004). H, Potential binding motifs present in the promoter regions of all melanogenesis-related genes are marked with red lines. Blue triangles represent the start of coding regions of these genes. The diagram was drawn using Illustrator for Biological Sequencing. Scale bar is shown at the bottom. 
SsSMR1 does not regulate these components. Thus, SsSMR1 appears to mainly regulate melanin biosynthesis genes. Interestingly, like in $B$. cinerea, some of these key melanin biosynthesis genes are encoded in tandem (Fig. 7E and F). SsPKS12 and SSSMRI are encoded in a tail-to-tail orientation on chromosome 12, along with the loosely linked SsLAC1 (sscle_12g090390). SsSCD1, SsBRN2, and SsPKS13 are all encoded in the same cluster on chromosome 3.

\section{DISCUSSION}

To unbiasedly identify genes involved in a particular biological process, forward genetic approaches are often utilized in model organisms by screening populations after random mutagenesis. For most researchers, their first choices of mutagen are chemicals, such as ethyl methanesulfonate (EMS), as they can be easily applied with predictable nucleotide change consequences. Although we initially tested EMS on ascospores of S. sclerotiorum, the mutagenesis procedure failed as the protocol was too harsh for the fungal spores and difficult on the experimenter. Instead, UV mutagenesis, which is less laborious and more efficient compared with T-DNA insertional mutagenesis, proved to be highly effective for S. sclerotiorum. UV light has strong genotoxic effects that produces DNA damage, usually causing single nucleotide changes (Ikehata and Ono 2011). These mutations can be confidently captured by our NGS analysis pipeline.

Traditionally, following a mutant screen, positional cloning is done to map and identify mutated genes responsible for the mutant phenotypes. Mapping serves to reduce the genomic region for candidate identification. For example, in Arabidopsis EMS mutants, there are usually over 500 mutations throughout the genome in each mutagenized line. Mapping reduces the candidate mutations to less than five to ten in a narrow chromosomal region. However, genetic mapping is not feasible for mutants obtained from a homothallic fungus such as S. sclerotiorum that has not been shown to outcross in the lab. It is also not feasible for fungi with many nuclei in their sexual or asexual spores. Fortunately, based on our NGS analysis from the current mutants obtained from ascospore mutagenesis, the number of significant mutations in each mutant line is usually between 1 and 10. A large number of synonymous mutations or mutations in introns and untranslated regions can be initially excluded using bioinformatics analysis. The small number of more meaningful mutations is likely due to the haploid, binucleate nature of ascospores used for mutagenesis and the high relative dosage of UV we applied; ascospores are easily killed when an essential gene is mutated.

Due to the low mutation rate in each mutant, one disadvantage of our approach is the need to screen a large population. However, as most microorganisms can be easily manipulated in the lab with 96-well plates, large populations can usually be handled efficiently. From our population of 40,000, we identified two independent alleles of Sssmrl, suggesting that a similar sized population should already contain mutations in almost all genes of this organism (which possesses around 11,130 coding genes). As exemplified here, screening a large population is also helpful in narrowing down candidates, as independent alleles can harbor different mutations in the same gene, accelerating gene discovery.

Our Sssmrl mutants are useful tools for answering some of the Sclerotinia melanin-related questions. Many fungi produce melanin to protect themselves in adverse environments or against microbial degradation (Bell and Wheeler 1986; Butler and Day 1998). From our screen, we identified three melanization-deficient mutants. Discolored sclerotia isolates have been reported in $B$. cinerea (Zhou et al. 2018). Our study of pnk and M98 mutants facilitated the identification of the sclerotia melanin biosynthesis pathway in this fungus. There are various types of melanin synthesized by species-specific pathways within the fungal kingdom (Toledo et al. 2017). However, the most-studied and prevalent fungal melanin is DHN melanin, produced via a polyketide pathway. Lazarovits et al. (2000) tested whether S. sclerotiorum produces DHN melanin by using tricyclazole, which specifically inhibits the reductases involved in the DHN melanin biosynthesis pathway. This is further supported by reverse genetic evidence provided by Liang et al. (2017), who found that the disruption of two DHN melanin pathway genes, SCD1 and THR1, did not alter pathogenicity but resulted in slower growth, delayed sclerotial formation, reduced sclerotial numbers, and decreased resistance to UV irradiation in S. sclerotiorum. These two mutants displayed brown-colored sclerotia due to a later blockage of melanin synthesis. In agreement, our study showed that disruption of SSSMRI leads to global reduction of melanin biosynthesis pathway genes, resulting in pink sclerotia, increased sensitivity to UV light and toxic chemicals, smoother sclerotia surface, and reduced formation of apothecia. These results confirm that melanin is important for carpogenic growth and survival of $S$. sclerotiorum under bleach treatment or UV irradiation. However, melanin does not seem to be required for its vegetative growth and virulence.

BcSMR1 and SsSMR1 share $87 \%$ amino acid identity, and they may exert the same function in the two closely related fungi. A previous study showed that BcSMR1 only regulates the expression levels of its adjacent PKS-encoding gene, since genes in secondary metabolites biosynthesis pathways are often physically linked (Schumacher 2016). However, our RNA-seq data demonstrate that most of DHN melanogenic genes are downregulated in Sssmrl-1 background during sclerotial development, including the genes not clustered with SSSMR1, suggesting that SsSMR1 likely serves a more global role to regulate the expression of genes involved in melanogenesis. Strikingly, a specific "(T/G)CTCC(A/T/G)" element is detected in the promoter regions of all genes mentioned in the hypothetic melanin biosynthesis model (Fig. 7G and $\mathrm{H}$ ), which is consistent with a previous study on the DNA-binding activity of the SsSMR1 ortholog of a yeast-like fungus, Aureobasidium melanogenum (Jiang et al. 2020). Besides, such a conserved element is not observed in the promoter region of $S S S M R 1$, supporting our conclusion that SsSMR1 does not have autoregulation activity. From our current study, melanin biosynthesis is not affected in compound appressoria. As disruption of SsPKS13 blocks melanin accumulation in compound appressoria ( $\mathrm{Li}$ et al. 2018), it is possible that SsSMR1 predominately functions at specific growth stages including vegetative growth, sclerotium development, and apothecium formation but not during appressoria development. It is also possible that other regulators are functioning independently or in concert with SsSMR1 during appressoria maturation. Together, these analyses indicate that SsSMR1 serves as a master TF regulator of melanin biosynthesis during stages of the Sclerotinia life cycle, and it may directly bind to these elements existing in the promoter regions of key melanin biosynthetic enzymes to stimulate their expression.

In some plant-pathogenic fungi, such as M. oryzae (Howard and Ferrari 1989), C. lagenarium (Kubo et al. 1987), and Verticillium dahliae (Tokousbalides and Sisler 1979), melanin contributes to pathogenicity by influencing the development of penetration appressoria structures. However, in other fungi, such as Alternaria alternata (Thomma 2003), melanin production might not be essential for fungal pathogenicity. In our study, sclerotial melanization pathway is not needed for virulence of $S$. sclerotiorum or melanization of appressoria, as revealed through the analysis of the melanin-deficient mutants. This is consistent with previous report that different pathways are contributing to melanization of sclerotia and appressoria melanization does not 
seem to be needed for virulence (Wang and Rollins 2021). This could be due to the different appressoria structure of S. sclerotiorum, which may not need DHN melanin to enforce host entry.

In conclusion, our study establishes a pipeline for effective screening of mutants with phenotypes of interest and subsequent cloning by NGS. From the initial screen, we successfully identified 32 mutants with mitotically stable morphological phenotypes. Notably, many of these phenotypes have not been reported before in S. sclerotiorum, demonstrating the potential of this pipeline for novel gene discovery. Furthermore, detailed analysis of three melanization-deficient mutants exemplifies the feasibility of the whole pipeline, which could be used theoretically in many non-model eukaryotic organisms to isolate genes involved in particular biological processes.

\section{MATERIALS AND METHODS}

\section{Fungal strains and culture conditions.}

The WT strain S. sclerotiorum 1980 was provided by J. Rollins at the University of Florida. Fungal cultures were maintained on PDA (Shanghai Bio-Way Technology) medium at room temperature and were stored on PDA slants at $4^{\circ} \mathrm{C}$ or as sclerotia. For transformant screening, hygromycin B (Sigma) was added to a final concentration of $50 \mu \mathrm{g} / \mathrm{ml}$. Bacteria used in this study were grown in Luria-Bertani (Bio Basic) medium.

\section{UV mutagenesis.}

Ascospores were generated from sclerotia of WT strain $S$. sclerotiorum 1980 that were germinated carpogenically as described (Athukorala et al. 2010). Ascospores were stored on tin foil at $4^{\circ} \mathrm{C}$ in a desiccant in the dark. Ascospores were then resuspended and diluted with sterilized PDB (Shanghai Bio-Way Technology) to $10^{4}$ spores per milliliter. Spore suspension $(300 \mu \mathrm{l})$ was evenly spread on PDA plates (90 mm diameter) and dried. Then these plates were irradiated under UV light (TL-2000 Ultraviolet Translinker) for $15 \mathrm{~s}$, with an energy strength of $9,000 \mathrm{~mJ} / \mathrm{cm}^{2}$. The plates were incubated at room temperature for 2 days, until growing fungal colonies were visible, after which the colonies were individually picked and transferred to a 96-well plate by sterile toothpicks. Each well of the 96-well plate contained $150 \mu$ l of PDA. Mutants with desired phenotypes were visually identified in around 7 days and were transferred onto fresh PDA plates. The plates were incubated for 2 weeks to confirm the morphological defects.

\section{Genomic DNA extraction of $S$. sclerotiorum mutants for NGS.}

Actively growing fungal mycelia cultured in PDB for 5 to 7 days were harvested and ground to a fine powder in liquid nitrogen. The cetyltrimethylammonium bromide (CTAB) method was used to extract genomic DNA from the mutants (Cubero et al. 1999). The crudely extracted DNA was then purified with the DNeasy Plant Maxi kit (Li et al. 2002) before samples were sent for NGS with a commercial service (Novogene, Bioinformatics Technology Co. Ltd.). DNA degradation and contamination were monitored on $1 \%$ agarose gels. Paired-end library preparation, DNA-seq, and read quality control of samples were completed by Novogene, using Illumina sequencer NovaSeq 6000, resulting in 14 to 17 million clean reads per sample. Clean reads were aligned to the $S$. sclerotiorum genome (ASM185786v1).

\section{NGS data analysis.}

The sequence reads from NGS were mapped to the reference genome of WT strain S. sclerotiorum 1980 by BWA-MEM (Li 2013). Mutation sites were identified by SAMtools with default parameters (Li et al. 2009). Annotation of the mutations was performed with germline short variant discovery (SNPs + Indels) based on GATK best practices (Auwera et al. 2013). False mutations in repetitive sequences were manually removed as previously described (Huang et al. 2019).

\section{Sanger sequencing of SsSMR1 in pnk2.}

Full-length SSSMRI was amplified with SSSMRI-F and SsSMRl-R primers and genomic DNA of pnk2 isolated by CTAB method was used as template. A 3,376-bp PCR product was then sequenced using five primers (SsSMR1-Seq-1F-5R) by Novogene in China. All primers are listed in Supplementary Table S4.

\section{Target gene knockout and complementation.}

The split marker method was used to generate SSSMRI gene replacement constructs (Catlett et al. 2003). In brief, 1,394-bp upstream and 1,223-bp downstream flanking fragments of SSSMRI were amplified with the primer pairs $S S S M R I / 1 \mathrm{~F}-2 \mathrm{R}$ and $S S S M R 1 /$ $3 F-4 R$, respectively, and were fused with $h p h$ fragments amplified with the primers $\mathrm{HYG} / \mathrm{F}-\mathrm{HYG} / \mathrm{R}$ from $p C H-E F-1$ plasmid (shared by D. Jiang from Huazhong Agricultural University in China) (Yu et al. 2004). All primers used for PCR are listed in Supplementary Table S4. The amplified PCR products were cotransformed into protoplasts of WT as described (Yu et al. 2004). Transformants were selected on fresh PDA plates supplemented with hygromycin B. The individual germinating transformants were transferred onto new PDA plates with hygromycin B. Fresh mycelia were used to perform colony PCR with two pairs of primers, 7F, H855-R and H855-F, 8R (Supplementary Table S4), to confirm the insertion of hygromycin gene. Positive transformants were inoculated onto new PDA plates with hygromycin B by hyphal tip transfer. Two more rounds of the same colony PCR and selection were conducted to accumulate more nuclei with hygromycin insertions. Then the transformants were cultured in PDB for 2 days. The fresh mycelia mat was used to produce protoplasts as mentioned above. The resulting protoplasts were diluted with PDB supplemented with hygromycin B to $10^{3}$ protoplasts per milliliter. A 1-ml suspension was evenly spread on PDA plates supplemented with hygromycin B and was dried. The germinating colonies were transferred onto individual PDA plates with hygromycin $\mathrm{B}$. Then the CTAB method was used to extract DNA from transformants, which were later used as template to conduct PCR with four pairs of primers as shown in Figure 4B. The transformants with the target gene being homogeneously knocked out were chosen for further experiments.

For transgene complementation, genomic SsSMRl was PCRamplified and was cloned into $p C H-E F-1$. The full-length genomic sequence of SSSMRI containing a 1.323-bp upstream region from the start codon was amplified, using specific primers (Supplementary Table S4). This fragment was digested, using restriction enzyme $X m a \mathrm{I}$, and was ligated into $p C H-E F-1$ vector to generate the $p C H$-SsSMRI construct. The above construct was introduced into Sssmr1-1, using a previously described protocol (Yu et al. 2012). Transformants were selected and were purified on fresh PDA plates supplemented with hygromycin B.

\section{Colony morphology and growth rate determination.}

WT strain, knockout mutants, and complementing transformants were grown on PDA plate for three to four days. Then, mycelial agar disks were taken from the colony margin with a sterilized hole punch ( $5 \mathrm{~mm}$ diameter), were transferred to the center of fresh PDA plates ( $90 \mathrm{~mm}$ diameter), and were incubated at room temperature. The colony diameter was measured every day until mycelia reached the edge of the PDA plate. The images of colony morphology were taken 14 days postinoculation (dpi).

\section{Plant infection assay.}

Pathogenicity of the strains was tested on detached leaves of Arabidopsis thaliana (ecotype Col-0) that were 4 weeks old. Mycelial 
agar plugs of $2 \mathrm{~mm}$ in diameter were inoculated on detached leaves placed on moistened paper towels in Petri dishes $(90 \mathrm{~mm}$ diameter), with one agar plug per leaf and five leaves per genotype. oahl is an oxalate-deficient mutant that exhibits significantly attenuated virulence (Liang et al. 2015) and served as the negative control. Photos were taken at 24 and $48 \mathrm{~h}$ postinoculation. The pathogenicity test was repeated three times with similar results.

\section{Apothecia and compound appressoria formation.}

Equal numbers of sclerotia were collected from WT, Sssmr1-1, and M98 and were used to generate apothecia as described (Athukorala et al. 2010). For compound appressoria formation, fresh mycelial plugs $(5 \mathrm{~mm}$ diameter) with growing hyphal tips were placed on glass slides and were incubated in a humidity chamber for 2 to 3 days. Then compound appressoria were observed and photographed under a microscope.

\section{Bleach treatment and UV irradiation assay on sclerotia.}

For bleach treatment, sclerotia, formed from two-week-old PDA plates of WT, Sssmr1-1, and Sssmr1-3, were treated with $15 \%$ bleach (Cloromax) for 30, 60, and 90 min. For UV irradiation, sclerotia were irradiated under UV light (TL-2000 Ultraviolet Translinker, energy strength of $9,000 \mathrm{~mJ} / \mathrm{cm}^{2}$ ) for 10,20 , and $30 \mathrm{~min}$. For both assays, each treatment included 45 sclerotia from WT, Sssmr1-1, or Sssmr1-3. The treated sclerotia were then grown on PDA for 3 days and the germination rate was calculated by the number of germinated sclerotia divided by the total number of sclerotia treated. The assays were performed three times with similar results.

\section{RNA-seq analysis.}

WT and Sssmrl-1 were cultured on PDA plates (90 mm diameter) and were incubated in the dark at room temperature for 3 days. Actively growing mycelia were transferred into flasks with $200 \mathrm{ml}$ of fresh PDB media for an additional 4 to 9 days of growth in the dark at room temperature. Young mycelia were harvested from WT and Sssmr1-1 PDB cultures at 4 dpi, while old mycelia were collected at 9 dpi. Young and old mycelial samples were collected in three biological replicates. Total RNA was extracted using TRIzol reagent according to manufacturer protocol (Invitrogen). Then, RNA degradation and contamination were monitored on $1 \%$ agarose gels. RNA integrity was assessed using the RNA Nano 6000 assay kit of the Agilent Bioanalyzer 2100 system (Agilent Technologies).

Paired-end library preparation, RNA-seq, and read-quality control of samples were completed by Novogene using Illumina sequencers resulting in 20 to 23 million clean reads per sample. Clean reads were aligned to the $S$. sclerotiorum genome (ASM185786v1), using HISAT2 v2.2.0 on default parameters (Kim et al. 2019). SAMtools v1.10 was used to sort and index bam files ( $\mathrm{Li}$ et al. 2009). Gene transcript counts were generated using featureCounts from the Subread v2.0.1 package on default parameters (Liao et al. 2014). GO term enrichment analysis using Fisher's exact test was done using annotations generated from Blast2GO (Götz et al. 2008). The R package DESeq2 v1.28.1 was used to identify DEGs between samples (Love et al. 2014). Gene rows were reorganized using Plots generated using the ComplexHeatmap, UpSetR, and ggplot2 packages in R (Conway et al. 2017; Gu et al. 2016; Villanueva and Chen 2019).

\section{Statistical analysis.}

One-way analysis of variance followed by Tukey's post hoc test were performed. The Scheffé multiple comparison was applied for testing correction. Normality test for all data were done in SPSS. Statistical significance was indicated with asterisks. $P$ values are included in figure captions.

\section{ACKNOWLEDGMENTS}

We cordially thank D. Jiang (Huazhong Agricultural University) for sharing $p C H-E F-1$ plasmid, J. Rollins (University of Florida) for sharing wild-type S. sclerotiorum 1980 and the oah1 mutant. S. Shang (Department of Botany, University of British Columbia) is sincerely thanked for assistance with the NGS data analysis.

\section{LITERATURE CITED}

Athukorala, S. N. P., Fernando, W. G. D., Rashid, K. Y., and Kievit, T. 2010. The role of volatile and non-volatile antibiotics produced by Pseudomonas chlororaphis strain PA23 in its root colonization and control of Sclerotinia sclerotiorum. Biocontrol Sci. Technol. 20:875-890.

Auwera, G. A. V., Carnerio, M. O., Hartl, C., Poplin, R., Angel, G. Moonshine, A., Jordan, T., Shakir, K., Roazen, D., Thibault, J., Banks, E. Garimella, K. V., Altshuler, S., and DePristo, M. A. 2013. From FastQ data to high confidence variant calls: The Genome Analysis Toolkit best practices pipeline. Curr. Protoc. Bioinformatics 11: 11.10.1-11.10.33.

Bailey, T. L., Boden, M., Buske, F. A., Frith, M., Grant, C. E., Clementi, L., Ren, J., Li, W. W., and Noble, W. S. 2009. MEME SUITE: Tools for motif discovery and searching. Nucleic Acids Res. 37:W202W208.

Bell, A. A., and Wheeler, M. H. 1986. Biosynthesis and functions of fungal melanins. Annu. Rev. Phytopathol. 24:411-451.

Bhadauria, V., Banniza, S., Wei, Y., and Peng, Y. L. 2009. Reverse genetics for functional genomics of phytopathogenic fungi and oomycetes. Comp. Funct. Genomics 2009:380719.

Boland, G. J., and Hall, R. 1994. Index of plant hosts of Sclerotinia sclerotiorum. Can. J. Plant Pathol. 16:93-108.

Bolton, M. D., Thomma, B. P. H. J., and Nelson, B. D. 2006. Sclerotinia sclerotiorum (Lib.) de Bary: Biology and molecular traits of a cosmopolitan pathogen. Mol. Plant Pathol. 7:1-16.

Butler, M. J., and Day, A. W. 1998. Fungal melanins: A review. Can. J. Microbiol. 44:1115-1136.

Butler, M. J., Gardiner, R. B., and Day, A. W. 2009. Melanin synthesis by Sclerotinia sclerotiorum. Mycologia 101:296-304.

Catlett, N. L., Lee, B. N., Yoder, O. C., and Gillian, T. B. 2003. Splitmarker recombination for efficient targeted deletion of fungal genes. Fungal Genet. Rep. 50:9-11.

Conway, J. R., Lex, A., and Gehlenborg, N. 2017. UpSetR: An R package for the visualization of intersecting sets and their properties. Bioinformatics 33:2938-2940.

Crooks, G. E., Hon, G., Chandonia, J. M., and Brenner, S. E. 2004 WebLogo: A sequence logo generator. Genome Res. 14:1188-1190.

Cubero, O. F., Crespo, A., Fatehi, J., and Bridge, P. D. 1999. DNA extraction and PCR amplification method suitable for fresh, herbarium-stored, lichenized, and other fungi. Plant Syst. Evol. 249:243-249.

Doehlemann, G., Ökmen, B., Zhu, W. J., and Sharon, A. 1987. Plant pathogenic fungi. Mycologia 79:919.

Fisher, M. C., Henk, D. A., Briggs, C. J., Brownstein, J. S., Madoff, L. C., McCraw, S. L., and Gurr, S. J. 2012. Emerging fungal threats to animal, plant and ecosystem health. Nature 484:186-194.

Fry, W. 2008. Phytophthora infestans: The plant (and $R$ gene) destroyer. Mol. Plant Pathol. 9:385-402.

Godoy, G., Steadman, J. R., Dickman, M. B., and Dam, R. 1990. Use of mutants to demonstrate the role of oxalic acid in pathogenicity of Sclerotinia sclerotiorum on Phaseolus vulgaris. Physiol. Mol. Plant Pathol. 37:179-191.

Götz, S., García-Gómez, J. M., Terol, J., Williams, T. D., Nagaraj, S. H., Nueda, M. J., Robles, M., Talón, M., Dopazo, J., and Conesa, A 2008. High-throughput functional annotation and data mining with the Blast2GO suite. Nucleic Acids Res. 36:3420-3435.

Gu, Z., Eils, R., and Schlesner, M. 2016. Complex heatmaps reveal patterns and correlations in multidimensional genomic data. Bioinformatics 32:2847-2849.

Howard, R. J., and Ferrari, M. A. 1989. Role of melanin in appressorium function. Exp. Mycol. 13:403-418.

Huang, J., Sun, Y., Orduna, A. R., Jetter, R., and Li, X. 2019. The Mediator kinase module serves as a positive regulator of salicylic acid accumulation and systemic acquired resistance. Plant J. 98:842-852.

Ikehata, H., and Ono, T. 2011. The mechanisms of UV mutagenesis. J. Radiat. Res. (Tokyo) 52:115-125.

Jiang, H., Chi, Z., Liu, G. L., Hu, Z., Zhao, S. Z., and Chi, Z. M. 2020. Melanin biosynthesis in the desert-derived Aureobasidium melanogenum XJ5-1 is controlled mainly by the CWI signal pathway via a transcriptional activator Cmr1. Curr. Genet. 66:173-185. 
Kim, D., Paggi, J. M., Park, C., Bennett, C., and Salzberg, S. L. 2019. Graph-based genome alignment and genotyping with HISAT2 and HISAT-genotype. Nat. Biotechnol. 37:907-915.

Kubo, Y., Furusawa, I., and Shishiyama, J. 1987. Relation between pigment intensity and penetrating ability in appressoria of Colletotrichum lagenarium. Can. J. Microbiol. 33:870-873.

Lazarovits, G., Starratt, A. N., and Huang, H. C. 2000. The effect of tricyclazole and culture medium on production of the melanin precursor 1,8-dihydroxynaphthalene by Sclerotinia sclerotiorum isolate SS7. Pestic. Biochem. Physiol. 67:54-62.

Li, H. 2013. Aligning sequence reads, clone sequences and assembly contigs with BWA-MEM. arXiv 1303.399700

Li, H., Handsaker, B., Wysoker, A., Fennell, T., Ruan, J., Homer, N., Marth, G., Abecasis, G., Durbin, R., and 1000 Genome Project Data Processing Subgroup. 2009. The Sequence Alignment/Map format and SAMtools. Bioinformatics 25:2078-2079.

Li, J., Zhang, Y., Zhang, Y., Yu, P. L., Pan, H., and Rollins, J. A. 2018. Introduction of large sequence inserts by CRISPR-Cas9 to create pathogenicity mutants in multinucleate filamentous pathogen Sclerotinia sclerotiorum. MBio 9:e00567.

Li, X., Lassner, M., and Zhang, Y. 2002. Deleteagene: A fast neutron deletion mutagenesis-based gene knockout system for plants. Comp. Funct. Genomics 3:158-160.

Liang, X., Liberti, D., Li, M., Kim, Y. T., Hutchens, A., Wilson, R., and Rollins, J. A. 2015. Oxaloacetate acetylhydrolase gene mutants of Sclerotinia sclerotiorum do not accumulate oxalic acid, but do produce limited lesions on host plants. Mol. Plant Pathol. 16:559-571.

Liang, X., and Rollins, J. A. 2018. Mechanisms of broad host range necrotrophic pathogenesis in Sclerotinia sclerotiorum. Phytopathology 108:1128-1140.

Liang, Y., Xiong, W., Steinkellner, S., and Feng, J. 2017. Deficiency of the melanin biosynthesis genes SCDI and THRI affects sclerotial development and vegetative growth, but not pathogenicity in Sclerotinia sclerotiorum. Mol. Plant Pathol. 19:1444-1453.

Liao, Y., Smyth, G. K., and Shi, W. 2014. featureCounts: An efficient general purpose program for assigning sequence reads to genomic features. Bioinformatics 30:923-930.

Liu, W., Xie, Y., Ma, J., Luo, X., Nie, P., Zuo, Z., Lahrmann, U., Zhao, Q., Zheng, Y., Zhao, Y., Xue, Y., and Ren, J. 2015. IBS: An illustrator for the presentation and visualization of biological sequences. Bioinformatics 31:3359-3361.

Love, M. I., Huber, W., and Anders, S. 2014. Moderated estimation of fold change and dispersion for RNA-seq data with DESeq2. Genome Biol. 15:550.

Miller, R. V., Ford, E. J., and Sands, D. C. 1989. A nonsclerotial pathogenic mutant of Sclerotinia sclerotiorum. Can. J. Microbiol. 35:517-520.

Mullins, E. D., and Kang, S. 2001. Transformation: A tool for studying fungal pathogens of plants. Cell. Mol. Life Sci. 58:2043-2052.

Saitoh, Y., Izumitsu, K., Morita, A., and Tanaka, C. 2010. A coppertransporting ATPase BcCCC2 is necessary for pathogenicity of Botrytis cinerea. Mol. Genet. Genomics 284:33-43.

Schumacher, J. 2016. DHN melanin biosynthesis in the plant pathogenic fungus Botrytis cinerea is based on two developmentally regulated key enzyme (PKS)-encoding genes. Mol. Microbiol. 99:729-748.

Schumacher, J., Simon, A., Cohrs, K. C., Traeger, S., Porquier, A., Dalmais, B., Viaud, M., and Tudzynski, B. 2015. The VELVET complex in the gray mold fungus Botrytis cinerea: Impact of BcLAE1 on differentiation, secondary metabolism, and virulence. Mol. PlantMicrobe Interact 28:659-674.

Strange, R. N., and Scott, P. R. 2005. Plant disease: A threat to global food security. Annu. Rev. Phytopathol. 43:83-116.

Sturz, A. V., Carter, M. R., and Johnston, H. W. 1997. A review of plant disease, pathogen interactions and microbial antagonism under conservation tillage in temperate humid agriculture. Soil Tillage Res. 41:169-189.

Thomma, B. P. H. J. 2003. Alternaria spp.: From general saprophyte to specific parasite. Mol. Plant Pathol. 4:225-236.

Tokousbalides, M. C., and Sisler, H. D. 1979. Site of inhibition by tricyclazole in the melanin biosynthetic pathway of Verticillium dahliae. Pestic. Biochem. Physiol. 11:64-73.

Toledo, A. V., Franco, M. E. E., Lopez, S. M. Y., Troncozo, M. I., Saparrat, M. C. N., and Balatti, P. A. 2017. Melanins in fungi: Types, localization and putative biological roles. Physiol. Mol. Plant Pathol. 99:2-6.

Tsuji, G., Fujikawa, J., Ishida, H., Horino, O., and Kubo, Y. 2001. Laccase Gene LAC1 of Colletotrichum lagenarium is not essential for melanin biosynthesis and pathogenicity. J. Gen. Plant Pathol. 67:182-190.

Ullstrup, A. J. 1972. The impacts of the southern corn leaf blight epidemics of 1970-1971. Annu. Rev. Phytopathol. 10:37-50.

Villanueva, R. A. M., and Chen, Z. J. 2019. ggplot2: Elegant graphics for data analysis (2nd ed.). Measurement: Interdisciplinary Research and Perspectives 17:3, 160-167.

Wang, C., and Rollins, J. A. 2021. Efficient genome editing using endogenous U6 snRNA promoter-driven CRISPR/Cas9 sgRNA in Sclerotinia sclerotiorum. Fungal Genet. Biol. 154:103598.

Willetts, H. and Wong, J. J. 1980. The biology of Sclerotinia sclerotiorum, $S$. trifoliorum, and $S$. minor with emphasis on specific nomenclature. Bot. Rev. 46:101-165.

Williamson, B., Tudzynski, B., Tudzynski, P., and van Kan, J. A. 2007. Botrytis cinerea: The cause of grey mould disease. Mol. Plant Pathol. 8:561-580.

Xia, S., Xu, Y., Hoy, R., Zhang, J., Qin, L., and Li, X. 2020. The notorious soilborne pathogenic fungus sclerotinia sclerotiorum: An update on genes studied with mutant analysis. Pathogens 9:27.

$\mathrm{Xu}$, L., and Chen, W. 2013. Random T-DNA mutagenesis identifies a $\mathrm{Cu} / \mathrm{Zn}$ superoxide dismutase gene as a virulence factor of Sclerotinia sclerotiorum. Mol. Plant-Microbe Interact 26:431-441.

Yang, Q., Chen, Y., and Ma, Z. 2013. Involvement of BcVeA and $\mathrm{BcVelB}$ in regulating conidiation, pigmentation and virulence in Botrytis cinerea. Fungal Genet. Biol. 50:63-71.

Yu, J. H., Hamari, Z., Han, K. H., Seo, J. A., Reyes-Domínguez, Y., and Scazzocchio, C. 2004. Double-joint PCR: A PCR-based molecular tool for gene manipulations in filamentous fungi. Fungal Genet. Biol. 41: 973-981.

Yu, Y., Jiang, D., Xie, J., Cheng, J., Li, G., Yi, X., and Fu, Y. 2012 Ss-S12, a novel cell wall protein with PAN modules, is essential for sclerotial development and cellular integrity of Sclerotinia sclerotiorum. PLoS One 7:e34962.

Zhou, Y., Li, N., Yang, J., Yang, L., Wu, M., Chen, W., Li, G., and Zhang, J. 2018. Contrast between orange- and black-colored sclerotial isolates of Botrytis cinerea: Melanogenesis and ecological fitness. Plant Dis. 102:428-436.

Zhou, Y., Yang, L., Wu, M., Chen, W., Li, G., and Zhang, J. 2017. A single-nucleotide deletion in the transcription factor gene bcsmr causes sclerotial-melanogenesis deficiency in Botrytis cinerea. Front. Microbiol. 8:2492. 